\title{
Mathematical Programming Based on Sufficient Optimality Conditions and Higher Order Exponential Type Generalized Invexities
}

\author{
Ram U. Verma * \\ Department of Mathematics, Texas State University, USA
}

Received: 27 March 2015; Accepted: 18 August 2015

Editor: Yiju Wang

\begin{abstract}
First, a class of comprehensive higher order exponential type generalized $B-(b, \rho, \eta, \omega, \theta, \tilde{p}, \tilde{r}, \tilde{s})$-invexities is introduced, which encompasses most of the existing generalized invexity concepts in the literature, including the Antczak type first order $B-(b, \eta, \tilde{p}, \tilde{r})$-invexities as well as the Zalmai type $(\alpha, \beta, \gamma, \eta, \rho, \theta)$-invexities, and then a wide range of parametrically sufficient optimality conditions leading to the solvability for discrete minimax fractional programming problems are established with some other related results. To the best of our knowledge, the obtained results are new and general in nature relating the investigations on generalized higher order exponential type invexities.
\end{abstract}

Keywords: Generalized higher order invexity, Minimax fractional programming, optimal solutions, sufficient optimality conditions

AMS 2010 subject classifications: 90C30, 90C32, 90C34

DOI: $10.19139 /$ soic.v3i3.139

\section{Introduction}

Recently, Zalmai [41], in a series of publications based on the work of Antczak [1, 2, 3], generalized the exponential type of invexities and applied to a class of global parametric sufficient optimality criteria using various assumptions for semiinfinite discrete minimax fractional programming problems. Furthermore, Zalmai [41], applying certain suitable partitioning schemes investigated various sets of generalized parametric sufficient optimality results each of which is in fact a family of such results whose members can easily be identified by appropriate choices of certain sets and functions. Antczak [1,2,3] introduced and studied first order exponential type $B$ - $(p, r)$ invexities and applied investigating nonlinear mathematical programming problems, especially in [2] Antczak proved some optimality conditions for a class of generalized fractional programming problems involving $B$ - $(p, r)$ invex functions. This work was followed by developing various duality models relating to fractional programming problems in the literature. Verma [30] introduced the second order $(\Phi, \Psi, \rho, \eta, \theta)$-invexities to the context of parametric sufficient optimality conditions in semiinfinite discrete minimax fractional programming, while Zalmai and Zhang [42] have established a set of necessary efficiency conditions and a fairly large number of global nonparametric sufficient efficiency results under various frameworks for generalized $(\eta, \rho)$-invexity for semiinfinite discrete minimax fractional programming problems. There exists an enormous literature on generalized first order as well as second order invexities with applications. Verma [25] also developed a general framework for a class of $(\rho, \eta, \theta)$-invex functions to examine some parametric sufficient efficiency conditions for multiobjective

*Correspondence to: Email: verma99@msn.com

ISSN 2310-5070 (online) ISSN 2311-004X (print)

Copyright (C) 2015 International Academic Press 
fractional programming problems for weakly $\epsilon$-efficient solutions. Motivated by the recent advances on $B$ - $(p$, $r$ )-invexities and other generalizations to the context of multiobjective fractional programming problems, we first introduce the higher order exponential type $B-(b, \rho, \eta, \omega, \theta, \tilde{p}, \tilde{r}, \tilde{s})$-invexities - a major generalization to Antczak type $B$ - $(\tilde{p}, \tilde{r})$-invexities - well-explored and well-cited in the literature, second we establish some parametric sufficient efficiency conditions for multiobjective fractional programming to achieve optimal solutions to multiobjective fractional programming problems, and then we establish some generalized sufficiency results. The results established in this communication, not only generalize the results on Antczak type, Zalmai, and Zalmai and Zhang type first order invexities but also generalize the second order invexity results in general settings.

We consider under the general framework of the second order $B-(b, \rho, \eta, \omega, \theta, \tilde{p}, \tilde{r}, \tilde{s})$-invexities of functions, the following minimax fractional programming problem:

$$
\min _{x \in Q} \max _{1 \leq i \leq p} \frac{f_{i}(x)}{g_{i}(x)}
$$

subject to $x \in Q=\left\{x \in X: H_{j}(x) \leq 0, j \in\{1,2, \cdots, m\}\right\}$,

where $X$ is a nonempty open convex subset of $\mathbb{R}^{n}$ (n-dimensional Euclidean space), $f_{i}$ and $g_{i}$ for $i \in\{1, \cdots, p\}$ and $H_{j}$ for $j \in\{1, \cdots, m\}$ are real-valued functions defined on $X$ such that $f_{i}(x) \geq 0, g_{i}(x)>0$ for $i \in\{1, \cdots, p\}$ and for all $x \in Q$. Here $Q$ denotes the feasible set of $(\mathrm{P})$.

The general theory for semiinfinite programming problems offers a wide range of significant applications to several fields of research, including game theory, industrial engineering, mechanical engineering, statistical analysis, engineering design (including design of control systems, design of earthquakes-resistant structures, digital filters, and electronic circuits), random graphs, boundary value problems, wavelet analysis, environmental protection planning, decision and management sciences, optimal control problems, continuum mechanics, robotics, and data envelopment analysis. For more details, we refer the reader [1 - 44].

\section{Generalized second order invexities}

The general invexity theory has been investigated in several directions. We generalize the notion of the first order Antczak type $B-(\tilde{p}, \tilde{r})$-invexiies to the case of the second order $B-(b, \rho, \eta, \omega, \theta, \tilde{p}, \tilde{r}, \tilde{s})$-invexities. These notions of the second order invexity encompass most of the existing notions in the literature. Let $f$ be a twice continuously differentiable real-valued function defined on $X$.

\section{Definition 2.1}

The function $f$ is said to be second order $B-(b, \rho, \eta, \omega, \theta, \tilde{p}, \tilde{r}, \tilde{s})$-invex at $x^{*} \in X$ if there exist functions $\eta, \omega, \theta: X \times X \rightarrow \mathbb{R}^{n}$ and $b: X \times X \rightarrow[0, \infty)$, and real numbers $\tilde{r}, \tilde{p}$ and $\tilde{s}$ such that for all $x \in X$ and $z \in \mathbb{R}^{n}$,

$$
\begin{aligned}
& b\left(x, x^{*}\right)\left(\frac{1}{\tilde{r}}\left(e^{\tilde{r}\left[f(x)-f\left(x^{*}\right)\right]}-1\right)\right) \geq \frac{1}{\tilde{p}}\left(\left\langle\nabla f\left(x^{*}\right), e^{\tilde{p} \eta\left(x, x^{*}\right)}-\mathbf{1}\right\rangle\right) \\
&+ \frac{1}{\tilde{s}}\left(\frac{1}{2}\left\langle\nabla^{2} f\left(x^{*}\right) z, e^{\tilde{s} \omega\left(x, x^{*}\right)}-\mathbf{1}\right\rangle\right) \\
&+ \rho\left(x, x^{*}\right)\left\|\theta\left(x, x^{*}\right)\right\|^{2} \text { for } \tilde{p} \neq 0, \tilde{r} \neq 0 \text { and } \tilde{s} \neq 0, \\
&+\quad b\left(x, x^{*}\right)\left(\frac{1}{\tilde{r}}\left(e^{\tilde{r}\left[f(x)-f\left(x^{*}\right)\right]}-1\right)\right) \geq\left\langle\nabla f\left(x^{*}\right), \eta\left(x, x^{*}\right)\right\rangle+\frac{1}{2}\left\langle\omega\left(x, x^{*}\right), \nabla^{2} f\left(x^{*}\right) z\right\rangle \\
&+\quad \theta\left(x, x^{*}\right) \|^{2} \text { for } \tilde{p}=0, \tilde{s}=0 \text { and } \tilde{r} \neq 0,
\end{aligned}
$$




$$
\begin{gathered}
b\left(x, x^{*}\right)\left(\left[f(x)-f\left(x^{*}\right)\right]\right) \geq \frac{1}{\tilde{p}}\left(\left\langle\nabla f\left(x^{*}\right), e^{\tilde{p} \eta\left(x, x^{*}\right)}-\mathbf{1}\right\rangle\right) \\
+\frac{1}{\tilde{s}}\left(\frac{1}{2}\left\langle e^{\tilde{s} \omega\left(x, x^{*}\right)}-\mathbf{1}, \nabla^{2} f\left(x^{*}\right) z\right\rangle\right) \\
+\quad \rho\left(x, x^{*}\right)\left\|\theta\left(x, x^{*}\right)\right\|^{2} \text { for } \tilde{p} \neq 0, \tilde{s} \neq 0 \text { and } \tilde{r}=0, \\
b\left(x, x^{*}\right)\left(\left[f(x)-f\left(x^{*}\right)\right]\right) \geq\left\langle\nabla f\left(x^{*}\right), \eta\left(x, x^{*}\right)\right\rangle+\frac{1}{2}\left\langle\omega\left(x, x^{*}\right), \nabla^{2} f\left(x^{*}\right) z\right\rangle \\
+\quad \rho\left(x, x^{*}\right)\left\|\theta\left(x, x^{*}\right)\right\|^{2} \text { for } \tilde{p}=0, \tilde{r}=0 \text { and } \tilde{s}=0,
\end{gathered}
$$

where $\mathbf{1}=(1,1, \cdots, 1) \in \mathbb{R}^{n}$.

\section{Definition 2.2}

The function $f$ is said to be second order strictly $B-(b, \rho, \eta, \omega, \theta, \tilde{p}, \tilde{r}, \tilde{s})$-invex at $x^{*} \in X$ if there exist functions $\eta, \omega, \theta: X \times X \rightarrow \mathbb{R}^{n}$ and $b: X \times X \rightarrow[0, \infty)$, and real numbers $\tilde{r}, \tilde{s}$ and $\tilde{p}$ such that for all $x \in X$ and $z \in \mathbb{R}^{n}$,

$$
\begin{aligned}
& \quad b\left(x, x^{*}\right)\left(\frac{1}{\tilde{r}}\left(e^{\tilde{r}\left[f(x)-f\left(x^{*}\right)\right]}-1\right)\right)>\frac{1}{\tilde{p}}\left(\left\langle\nabla f\left(x^{*}\right), e^{\tilde{p} \eta\left(x, x^{*}\right)}-\mathbf{1}\right\rangle\right) \\
& +\quad \frac{1}{\tilde{s}}\left(\frac{1}{2}\left\langle e^{\tilde{s} \omega\left(x, x^{*}\right)}-\mathbf{1}, \nabla^{2} f\left(x^{*}\right) z\right\rangle\right) \\
& +\quad \rho\left(x, x^{*}\right)\left\|\theta\left(x, x^{*}\right)\right\|^{2} \text { for } \tilde{p} \neq 0, \tilde{s} \neq 0 \text { and } \tilde{r} \neq 0, \\
& b\left(x, x^{*}\right)\left(\frac{1}{\tilde{r}}\left(e^{\tilde{r}\left[f(x)-f\left(x^{*}\right)\right]}-1\right)\right)>\left\langle\nabla f\left(x^{*}\right), \eta\left(x, x^{*}\right)\right\rangle+\frac{1}{2}\left\langle\omega\left(x, x^{*}\right), \nabla^{2} f\left(x^{*}\right) z\right\rangle \\
& +\rho\left(x, x^{*}\right)\left\|\theta\left(x, x^{*}\right)\right\|^{2} \text { for } \tilde{p}=0, \tilde{s}=0 \text { and } \tilde{r} \neq 0, \\
& \quad b\left(x, x^{*}\right)\left(\left[f(x)-f\left(x^{*}\right)\right]\right)>\frac{1}{\tilde{p}}\left(\left\langle\nabla f\left(x^{*}\right), e^{\tilde{p} \eta\left(x, x^{*}\right)}-\mathbf{1}\right\rangle\right) \\
& +\quad\left(\frac{1}{2}\left\langle\omega\left(x, x^{*}\right), \nabla^{2} f\left(x^{*}\right) z\right\rangle\right) \\
& +\quad \rho\left(x, x^{*}\right)\left\|\theta\left(x, x^{*}\right)\right\|^{2} \text { for } \tilde{p} \neq 0, \tilde{s}=0 \text { and } \tilde{r}=0, \\
& \quad b\left(x, x^{*}\right)\left(\left[f(x)-f\left(x^{*}\right)\right]\right)>\left\langle\nabla f\left(x^{*}\right), \eta\left(x, x^{*}\right)\right\rangle+\frac{1}{2}\left\langle\omega\left(x, x^{*}\right), \nabla^{2} f\left(x^{*}\right) z\right\rangle \\
& +\quad \rho\left(x, x^{*}\right)\left\|\theta\left(x, x^{*}\right)\right\|^{2} \text { for } \tilde{p}=0, \tilde{r}=0 \text { and } \tilde{s}=0 .
\end{aligned}
$$

\section{Definition 2.3}

The function $f$ is said to be second order $B-(b, \rho, \eta, \omega, \theta, \tilde{p}, \tilde{r}, \tilde{s})$-pseudoinvex at $x^{*} \in X$ if there exist functions $\eta, \omega, \theta: X \times X \rightarrow \mathbb{R}^{n}$, and $b: X \times X \rightarrow[0, \infty)$, and real numbers $\tilde{r}, \tilde{s}$ and $\tilde{p}$ such that for all $x \in X$ and $z \in \mathbb{R}^{n}$,

$$
\begin{aligned}
& \frac{1}{\tilde{p}}\left(\left\langle\nabla f\left(x^{*}\right), e^{\tilde{p} \eta\left(x, x^{*}\right)}-\mathbf{1}\right\rangle\right) \\
&+ \frac{1}{\tilde{s}}\left(\frac{1}{2}\left\langle e^{\tilde{s} \omega\left(x, x^{*}\right)}-\mathbf{1}, \nabla^{2} f\left(x^{*}\right) z\right\rangle\right)+\rho\left(x, x^{*}\right)\left\|\theta\left(x, x^{*}\right)\right\|^{2} \geq 0 \\
& \Rightarrow b\left(x, x^{*}\right)\left(\frac{1}{\tilde{r}}\left(e^{\tilde{r}\left[f(x)-f\left(x^{*}\right)\right]}-1\right)\right) \geq 0 \text { for } \tilde{p} \neq 0, \tilde{r} \neq 0 \text { and } \tilde{s} \neq 0, \\
&\left\langle\nabla f\left(x^{*}\right), \eta\left(x, x^{*}\right)\right\rangle+\frac{1}{2}\left\langle\omega\left(x, x^{*}\right), \nabla^{2} f\left(x^{*}\right) z\right\rangle+\rho\left(x, x^{*}\right)\left\|\theta\left(x, x^{*}\right)\right\|^{2} \geq 0 \\
& \Rightarrow \quad b\left(x, x^{*}\right)\left(\frac{1}{\tilde{r}}\left(e^{\tilde{r}\left[f(x)-f\left(x^{*}\right)\right]}-1\right)\right) \geq 0 \text { for } \tilde{p}=0, \tilde{s}=0 \text { and } \tilde{r} \neq 0,
\end{aligned}
$$




$$
\begin{aligned}
& \frac{1}{\tilde{p}}\left(\left\langle\nabla f\left(x^{*}\right), e^{\tilde{p} \eta\left(x, x^{*}\right)}-\mathbf{1}\right\rangle\right) \\
+ & \frac{1}{\tilde{s}}\left(\frac{1}{2}\left\langle e^{\tilde{s} \omega\left(x, x^{*}\right)}-\mathbf{1}, \nabla^{2} f\left(x^{*}\right) z\right\rangle\right)+\rho\left(x, x^{*}\right)\left\|\theta\left(x, x^{*}\right)\right\|^{2} \geq 0 \\
\Rightarrow \quad & b\left(x, x^{*}\right)\left(\left[f(x)-f\left(x^{*}\right)\right]\right) \geq 0 \text { for } \tilde{p} \neq 0, \tilde{s} \neq 0 \text { and } \tilde{r}=0, \\
& \left\langle\nabla f\left(x^{*}\right), \eta\left(x, x^{*}\right)\right\rangle+\frac{1}{2}\left\langle\omega\left(x, x^{*}\right), \nabla^{2} f\left(x^{*}\right) z\right\rangle \\
+\quad & \rho\left(x, x^{*}\right)\left\|\theta\left(x, x^{*}\right)\right\|^{2} \geq 0 \\
\Rightarrow & b\left(x, x^{*}\right)\left(\left[f(x)-f\left(x^{*}\right)\right]\right) \geq 0 \text { for } \tilde{p}=0, \tilde{r}=0, \tilde{s}=0 .
\end{aligned}
$$

\section{Definition 2.4}

The function $f$ is said to be second order strictly $B-(b, \rho, \eta, \omega, \theta, \tilde{p}, \tilde{r}, \tilde{s})$-pseudoinvex at $x^{*} \in X$ if there exist functions $\eta, \omega, \theta: X \times X \rightarrow \mathbb{R}^{n}$ and $b: X \times X \rightarrow[0, \infty)$, and real numbers $\tilde{r}$ and $\tilde{p}$ such that for all $x \in X$ and $z \in \mathbb{R}^{n}$,

$$
\begin{aligned}
& \frac{1}{\tilde{p}}\left(\left\langle\nabla f\left(x^{*}\right), e^{\tilde{p} \eta\left(x, x^{*}\right)}-\mathbf{1}\right\rangle\right) \\
&+ \frac{1}{\tilde{s}}\left(\frac{1}{2}\left\langle e^{\tilde{p} \omega\left(x, x^{*}\right)}-\mathbf{1}, \nabla^{2} f\left(x^{*}\right) z\right\rangle\right)+\rho\left(x, x^{*}\right)\left\|\theta\left(x, x^{*}\right)\right\|^{2} \geq 0 \\
& \Rightarrow \quad b\left(x, x^{*}\right)\left(\frac{1}{\tilde{r}}\left(e^{\tilde{r}\left[f(x)-f\left(x^{*}\right)\right]}-1\right)\right)>0 \text { for } \tilde{p} \neq 0, \tilde{r} \neq 0 \text { and } \tilde{s} \neq 0, \\
&\left\langle\nabla f\left(x^{*}\right), \eta\left(x, x^{*}\right)\right\rangle+\frac{1}{2}\left\langle\omega\left(x, x^{*}\right), \nabla^{2} f\left(x^{*}\right) z\right\rangle+\rho\left(x, x^{*}\right)\left\|\theta\left(x, x^{*}\right)\right\|^{2} \geq 0 \\
& \Rightarrow\left(x, x^{*}\right)\left(\frac{1}{\tilde{r}}\left(e^{\tilde{r}\left[f(x)-f\left(x^{*}\right)\right]}-1\right)\right)>0 \text { for } \tilde{p}=0, \tilde{s}=0 \text { and } \tilde{r} \neq 0, \\
& \quad \frac{1}{\tilde{p}}\left(\left\langle\nabla f\left(x^{*}\right), e^{\tilde{p} \eta\left(x, x^{*}\right)}-\mathbf{1}\right\rangle\right) \\
&+\quad \frac{1}{\tilde{s}}\left(\frac{1}{2}\left\langle e^{\tilde{s} \omega\left(x, x^{*}\right)}-\mathbf{1}, \nabla^{2} f\left(x^{*}\right) z\right\rangle\right)+\rho\left(x, x^{*}\right)\left\|\theta\left(x, x^{*}\right)\right\|^{2} \geq 0 \\
& \Rightarrow \quad b\left(x, x^{*}\right)\left(\left[f(x)-f\left(x^{*}\right)\right]\right)>0 \text { for } \tilde{p} \neq 0, \tilde{s} \neq 0 \text { and } \tilde{r}=0, \\
& \Rightarrow \quad b\left(x, x^{*}\right)\left(\left[f(x)-f\left(x^{*}\right)\right]\right)>0 \text { for } \tilde{p}=0, \tilde{s}=0 \text { and } \tilde{r}=0, \\
&\left\langle\nabla f\left(x^{*}\right), \eta\left(x, x^{*}\right)\right\rangle+\frac{1}{2}\left\langle\omega\left(x, x^{*}\right), \nabla^{2} f\left(x^{*}\right) z\right\rangle+\rho\left(x, x^{*}\right)\left\|\theta\left(x, x^{*}\right)\right\|^{2} \geq 0 \\
& \Rightarrow \quad
\end{aligned}
$$

equivalently,

$$
\begin{aligned}
& b\left(x, x^{*}\right)\left(\frac{1}{\tilde{r}}\left(e^{\tilde{r}\left[f(x)-f\left(x^{*}\right)\right]}-1\right)\right) \leq 0 \\
\Rightarrow & \frac{1}{\tilde{p}}\left(\left\langle\nabla f\left(x^{*}\right), e^{\tilde{p} \eta\left(x, x^{*}\right)}-\mathbf{1}\right\rangle\right)+\frac{1}{\tilde{s}}\left(\frac{1}{2}\left\langle e^{\tilde{p} \omega\left(x, x^{*}\right)}-\mathbf{1}, \nabla^{2} f\left(x^{*}\right) z\right\rangle\right) \\
+ & \rho\left(x, x^{*}\right)\left\|\theta\left(x, x^{*}\right)\right\|^{2}<0 \text { for } \tilde{p} \neq 0, \tilde{r} \neq 0 \text { and } \tilde{s} \neq 0 .
\end{aligned}
$$




\section{Definition 2.5}

The function $f$ is said to be second order prestrictly $B-(b, \rho, \eta, \omega, \theta, \tilde{p}, \tilde{r}, \tilde{s})$-pseudoinvex at $x^{*} \in X$ if there exist functions $\eta, \omega, \theta: X \times X \rightarrow \mathbb{R}^{n}$ and $b: X \times X \rightarrow[0, \infty)$, and real numbers $\tilde{r}$ and $\tilde{p}$ such that for all $x \in X$ and $z \in \mathbb{R}^{n}$,

$$
\begin{aligned}
& \frac{1}{\tilde{p}}\left(\left\langle\nabla f\left(x^{*}\right), e^{\tilde{p} \eta\left(x, x^{*}\right)}-\mathbf{1}\right\rangle\right. \\
+\quad & \frac{1}{\tilde{s}}\left(\frac{1}{2}\left\langle e^{\tilde{s} \omega\left(x, x^{*}\right)}-\mathbf{1}, \nabla^{2} f\left(x^{*}\right) z\right\rangle\right)+\rho\left(x, x^{*}\right)\left\|\theta\left(x, x^{*}\right)\right\|^{2}>0 \\
\Rightarrow \quad & b\left(x, x^{*}\right)\left(\frac{1}{\tilde{r}}\left(e^{\tilde{r}\left[f(x)-f\left(x^{*}\right)\right]}-1\right)\right) \geq 0 \text { for } \tilde{p} \neq 0, \tilde{s} \neq 0 \text { and } \tilde{r} \neq 0, \\
& \left\langle\nabla f\left(x^{*}\right), \eta\left(x, x^{*}\right)\right\rangle+\frac{1}{2}\left\langle z, \nabla^{2} f\left(x^{*}\right) z\right\rangle+\rho\left(x, x^{*}\right)\left\|\theta\left(x, x^{*}\right)\right\|^{2}>0 \\
\Rightarrow \quad & b\left(x, x^{*}\right)\left(\frac{1}{\tilde{r}}\left(e^{\tilde{r}\left[f(x)-f\left(x^{*}\right)\right]}-1\right)\right) \geq 0 \text { for } \tilde{p}=0, \tilde{s}=0 \text { and } \tilde{r} \neq 0, \\
& \\
& \frac{1}{\tilde{p}}\left(\left\langle\nabla f\left(x^{*}\right), e^{\tilde{p} \eta\left(x, x^{*}\right)}-\mathbf{1}\right\rangle\right. \\
+ & \frac{1}{2 \tilde{s}}\left\langle e^{\tilde{s} \omega\left(x, x^{*}\right)}-\mathbf{1}, \nabla^{2} f\left(x^{*}\right) z\right\rangle+\rho\left(x, x^{*}\right)\left\|\theta\left(x, x^{*}\right)\right\|^{2}>0 \\
\Rightarrow & b\left(x, x^{*}\right)\left(\left[f(x)-f\left(x^{*}\right)\right]\right) \geq 0 \text { for } \tilde{p} \neq 0, \tilde{s} \neq 0 \text { and } \tilde{r}=0, \\
\Rightarrow \quad b\left(x, x^{*}\right)\left(\left[f(x)-f\left(x^{*}\right)\right]\right) \geq 0 \text { for } \tilde{p}=0 \text { and } \tilde{r}=0 . & \left\langle\nabla f\left(x^{*}\right), \eta\left(x, x^{*}\right)\right\rangle+\frac{1}{2}\left\langle z, \nabla^{2} f\left(x^{*}\right) z\right\rangle+\rho\left(x, x^{*}\right)\left\|\theta\left(x, x^{*}\right)\right\|^{2}>0 \\
&
\end{aligned}
$$

\section{Definition 2.6}

The function $f$ is said to be second order $B-(b, \rho, \eta, \omega, \theta, \tilde{p}, \tilde{r}, \tilde{s})$-quasiinvex at $x^{*} \in X$ if there exist functions $\eta, \omega, \theta: X \times X \rightarrow \mathbb{R}^{n}$ and $b: X \times X \rightarrow[0, \infty)$, and real numbers $\tilde{r}, \tilde{s}$ and $\tilde{p}$ such that for all $x \in X$ and $z \in \mathbb{R}^{n}$,

$$
\begin{gathered}
b\left(x, x^{*}\right)\left(\frac{1}{\tilde{r}}\left(e^{\tilde{r}\left[f(x)-f\left(x^{*}\right)\right]}-1\right)\right) \leq 0 \\
\Rightarrow \quad \frac{1}{\tilde{p}}\left(\left\langle\nabla f\left(x^{*}\right), e^{\tilde{p} \eta\left(x, x^{*}\right)}-\mathbf{1}\right\rangle\right. \\
\left.+\frac{1}{2 \tilde{s}}\left\langle e^{\tilde{s} \omega\left(x, x^{*}\right)}-\mathbf{1}, \nabla^{2} f\left(x^{*}\right) z\right\rangle\right)+\rho\left(x, x^{*}\right)\left\|\theta\left(x, x^{*}\right)\right\|^{2} \leq 0 \text { for } \tilde{p} \neq 0, \tilde{s} \neq 0 \text { and } \tilde{r} \neq 0, \\
\quad b\left(x, x^{*}\right)\left(\frac{1}{\tilde{r}}\left(e^{\tilde{r}\left[f(x)-f\left(x^{*}\right)\right]}-1\right)\right) \leq 0 \\
\Rightarrow \quad\left\langle\nabla f\left(x^{*}\right), \eta\left(x, x^{*}\right)\right\rangle+\frac{1}{2}\left\langle\omega\left(x, x^{*}\right), \nabla^{2} f\left(x^{*}\right) z\right\rangle \\
+\quad \rho\left(x, x^{*}\right)\left\|\theta\left(x, x^{*}\right)\right\|^{2} \leq 0 ; \text { for } \tilde{p}=0, \tilde{s}=0 \text { and } \tilde{r} \neq 0,
\end{gathered}
$$




$$
\begin{aligned}
& b\left(x, x^{*}\right)\left(\left[f(x)-f\left(x^{*}\right)\right]\right) \leq 0 \\
\Rightarrow & \frac{1}{\tilde{p}}\left(\left\langle\nabla f\left(x^{*}\right), e^{\tilde{p} \eta\left(x, x^{*}\right)}-\mathbf{1}\right\rangle\right. \\
+ & \left.\frac{1}{2 \tilde{s}}\left\langle e^{\tilde{s} \omega\left(x, x^{*}\right)}-\mathbf{1}, \nabla^{2} f\left(x^{*}\right) z\right\rangle\right)+\rho\left(x, x^{*}\right)\left\|\theta\left(x, x^{*}\right)\right\|^{2} \leq 0
\end{aligned}
$$

for $\tilde{p} \neq 0, \tilde{s} \neq 0$ and $\tilde{r}=0$,

$$
\begin{aligned}
& b\left(x, x^{*}\right)\left(\left[f(x)-f\left(x^{*}\right)\right]\right) \leq 0 \\
\Rightarrow \quad & \left\langle\nabla f\left(x^{*}\right), \eta\left(x, x^{*}\right)\right\rangle+\frac{1}{2}\left\langle\omega\left(x, x^{*}\right), \nabla^{2} f\left(x^{*}\right) z\right\rangle \\
+\quad & \rho\left(x, x^{*}\right)\left\|\theta\left(x, x^{*}\right)\right\|^{2} \leq 0 \text { for } \tilde{p}=0 \text { and } \tilde{r}=0 .
\end{aligned}
$$

\section{Definition 2.7}

The function $f$ is said to be second order strictly $B-(b, \rho, \eta, \omega, \theta, \tilde{p}, \tilde{r}, \tilde{s})$-quasiinvex at $x^{*} \in X$ if there exist functions $\eta, \omega, \theta: X \times X \rightarrow \mathbb{R}^{n}$ and $b: X \times X \rightarrow[0, \infty)$, and real numbers $\tilde{r}, \tilde{s}$ and $\tilde{p}$ such that for all $x \in X$ and $z \in \mathbb{R}^{n}$,

$$
\begin{aligned}
& b\left(x, x^{*}\right)\left(\frac{1}{\tilde{r}}\left(e^{\tilde{r}\left[f(x)-f\left(x^{*}\right)\right]}-1\right)\right) \leq 0 \\
& \Rightarrow \frac{1}{\tilde{p}}\left(\left\langle\nabla f\left(x^{*}\right), e^{\tilde{p} \eta\left(x, x^{*}\right)}-\mathbf{1}\right\rangle\right. \\
& +\frac{1}{\tilde{s}}\left(\frac{1}{2}\left\langle e^{\tilde{s} \omega\left(x, x^{*}\right)}-\mathbf{1}, \nabla^{2} f\left(x^{*}\right) z\right\rangle\right)+\rho\left(x, x^{*}\right)\left\|\theta\left(x, x^{*}\right)\right\|^{2}<0 \text { for } \tilde{p} \neq 0, \tilde{s} \neq 0 \text { and } \tilde{r} \neq 0, \\
& b\left(x, x^{*}\right)\left(\frac{1}{\tilde{r}}\left(e^{\tilde{r}\left[f(x)-f\left(x^{*}\right)\right]}-1\right)\right) \leq 0 \\
& \Rightarrow\left\langle\nabla f\left(x^{*}\right), \eta\left(x, x^{*}\right)\right\rangle+\frac{1}{2}\left\langle\omega\left(x, x^{*}\right), \nabla^{2} f\left(x^{*}\right) z\right\rangle \\
& +\rho\left(x, x^{*}\right)\left\|\theta\left(x, x^{*}\right)\right\|^{2}<0 ; \text { for } \tilde{p}=0, \tilde{s}=0 \text { and } \tilde{r} \neq 0 \text {, } \\
& b\left(x, x^{*}\right)\left(\left[f(x)-f\left(x^{*}\right)\right]\right) \leq 0 \\
& \Rightarrow \frac{1}{\tilde{p}}\left(\left\langle\nabla f\left(x^{*}\right), e^{\tilde{p} \eta\left(x, x^{*}\right)}-\mathbf{1}\right\rangle\right) \\
& +\frac{1}{\tilde{s}}\left(\frac{1}{2}\left\langle e^{\tilde{s} \omega\left(x, x^{*}\right)}-\mathbf{1}, \nabla^{2} f\left(x^{*}\right) z\right\rangle\right)+\rho\left(x, x^{*}\right)\left\|\theta\left(x, x^{*}\right)\right\|^{2}<0 \text { for } \tilde{p} \neq 0, \tilde{s} \neq 0 \text { and } \tilde{r}=0, \\
& b\left(x, x^{*}\right)\left(\left[f(x)-f\left(x^{*}\right)\right]\right) \leq 0 \\
& \Rightarrow\left\langle\nabla f\left(x^{*}\right), \eta\left(x, x^{*}\right)\right\rangle+\frac{1}{2}\left\langle\omega\left(x, x^{*}\right), \nabla^{2} f\left(x^{*}\right) z\right\rangle \\
& +\rho\left(x, x^{*}\right)\left\|\theta\left(x, x^{*}\right)\right\|^{2}<0 \text { for } \tilde{p}=0, \tilde{s}=0 \text { and } \tilde{r}=0 .
\end{aligned}
$$




\section{Definition 2.8}

The function $f$ is said to be second order prestrictly $B-(b, \rho, \eta, \omega, \theta, \tilde{p}, \tilde{r}, \tilde{s})$-quasiinvex at $x^{*} \in X$ if there exist functions $\eta, \omega, \theta: X \times X \rightarrow \mathbb{R}^{n}$ and $b: X \times X \rightarrow[0, \infty)$, and real numbers $\tilde{r}$ and $\tilde{p}$ such that for all $x \in X$ and $z \in \mathbb{R}^{n}$,

$$
\begin{aligned}
& b\left(x, x^{*}\right)\left(\frac{1}{\tilde{r}}\left(e^{\tilde{r}\left[f(x)-f\left(x^{*}\right)\right]}-1\right)\right)<0 \\
& \Rightarrow \frac{1}{\tilde{p}}\left(\left\langle\nabla f\left(x^{*}\right), e^{\tilde{p} \eta\left(x, x^{*}\right)}-\mathbf{1}\right\rangle\right)+\frac{1}{2 \tilde{s}}\left(\left\langle e^{\tilde{s} \omega\left(x, x^{*}\right)}-\mathbf{1}, \nabla^{2} f\left(x^{*}\right) z\right\rangle\right. \\
& +\rho\left(x, x^{*}\right)\left\|\theta\left(x, x^{*}\right)\right\|^{2} \leq 0 \text { for } \tilde{p} \neq 0, \tilde{s} \neq 0 \text { and } \tilde{r} \neq 0, \\
& b\left(x, x^{*}\right)\left(\frac{1}{\tilde{r}}\left(e^{\tilde{r}\left[f(x)-f\left(x^{*}\right)\right]}-1\right)\right)<0 \\
& \Rightarrow\left\langle\nabla f\left(x^{*}\right), \eta\left(x, x^{*}\right)\right\rangle+\frac{1}{2}\left\langle\omega\left(x, x^{*}\right), \nabla^{2} f\left(x^{*}\right) z\right\rangle \\
& +\rho\left(x, x^{*}\right)\left\|\theta\left(x, x^{*}\right)\right\|^{2} \leq 0 ; \text { for } \tilde{p}=0, \tilde{s}=0 \text { and } \tilde{r} \neq 0 \text {, } \\
& b\left(x, x^{*}\right)\left(\left[f(x)-f\left(x^{*}\right)\right]\right)<0 \\
& \Rightarrow \frac{1}{\tilde{p}}\left(\left\langle\nabla f\left(x^{*}\right), e^{\tilde{p} \eta\left(x, x^{*}\right)}-\mathbf{1}\right\rangle\right) \\
& +\frac{1}{\tilde{s}}\left(\frac{1}{2}\left\langle e^{\tilde{s} \omega\left(x, x^{*}\right)}-\mathbf{1}, \nabla^{2} f\left(x^{*}\right) z\right\rangle\right) \\
& +\rho\left(x, x^{*}\right)\left\|\theta\left(x, x^{*}\right)\right\|^{2} \leq 0 \text { for } \tilde{p} \neq 0, \tilde{s} \neq 0 \text { and } \tilde{r}=0, \\
& b\left(x, x^{*}\right)\left(\left[f(x)-f\left(x^{*}\right)\right]\right)<0 \\
& \Rightarrow\left\langle\nabla f\left(x^{*}\right), \eta\left(x, x^{*}\right)\right\rangle \\
& +\frac{1}{2}\left\langle\omega\left(x, x^{*}\right), \nabla^{2} f\left(x^{*}\right) z\right\rangle+\rho\left(x, x^{*}\right)\left\|\theta\left(x, x^{*}\right)\right\|^{2} \leq 0 \text { for } \tilde{p}=0, \tilde{s}=0 \text { and } \tilde{r}=0,
\end{aligned}
$$

equivalently,

$$
\begin{aligned}
& \frac{1}{\tilde{p}}\left(\left\langle\nabla f\left(x^{*}\right), e^{\tilde{p} \eta\left(x, x^{*}\right)}-\mathbf{1}\right\rangle\right)+\frac{1}{2 \tilde{s}}\left(\left\langle e^{\tilde{s} \omega\left(x, x^{*}\right)}-\mathbf{1}, \nabla^{2} f\left(x^{*}\right) z\right\rangle\right) \\
+ & \rho\left(x, x^{*}\right)\left\|\theta\left(x, x^{*}\right)\right\|^{2}>0 \\
\Rightarrow & b\left(x, x^{*}\right)\left(\frac{1}{\tilde{r}}\left(e^{\tilde{r}\left[f(x)-f\left(x^{*}\right)\right]}-1\right)\right) \geq 0 \text { for } \tilde{p} \neq 0, \tilde{s} \neq 0 \text { and } \tilde{r} \neq 0 .
\end{aligned}
$$

Next, we present some examples which shall reflect the interrelationship among the basic definitions introduced (and applied) in this paper.

\section{Example 2.1}

The function $f$ is said to be second order $B-(b, \rho, \eta, \theta, \tilde{p}, \tilde{r}, \tilde{s})$-pseudoinvex with respect to $\eta$ and $b$ at $x^{*} \in X$ if there exist functions $\eta, \theta: X \times X \rightarrow \mathbb{R}^{n}$ and $b: X \times X \rightarrow[0, \infty)$, and real numbers $\tilde{r}, \tilde{s}$ and $\tilde{p}$ such that for all 
$x \in X$ and $z \in \mathbb{R}^{n}$,

$$
\begin{aligned}
& \frac{1}{\tilde{p}}\left(\left\langle\nabla f\left(x^{*}\right), e^{\tilde{p} \eta\left(x, x^{*}\right)}-\mathbf{1}\right\rangle\right) \\
+ & \frac{1}{\tilde{s}}\left(\frac{1}{2}\left\langle e^{\tilde{s} z}-\mathbf{1}, \nabla^{2} f\left(x^{*}\right) z\right\rangle\right)+\rho\left(x, x^{*}\right)\left\|\theta\left(x, x^{*}\right)\right\|^{2} \geq 0 \\
\Rightarrow & b\left(x, x^{*}\right)\left(\frac{1}{\tilde{r}}\left(e^{\tilde{r}\left[f(x)-f\left(x^{*}\right)\right]}-1\right)\right) \geq 0 \text { for } \tilde{p} \neq 0, \tilde{r} \neq 0 \text { and } \tilde{s} \neq 0 .
\end{aligned}
$$

\section{Example 2.2}

The function $f$ is said to be second order $B-(b, \rho, \eta, \theta, \tilde{p}, \tilde{r})$-pseudoinvex with respect to $\eta$ and $b$ at $x^{*} \in X$ if there exist functions $\eta, \theta: X \times X \rightarrow \mathbb{R}^{n}$ and $b: X \times X \rightarrow[0, \infty)$, and real numbers $\tilde{r}$ and $\tilde{p}$ such that for all $x \in X$ and $z \in \mathbb{R}^{n}$

$$
\begin{aligned}
& \frac{1}{\tilde{p}}\left(\left\langle\nabla f\left(x^{*}\right)+\frac{1}{2} \nabla^{2} f\left(x^{*}\right) z, e^{\tilde{p} \eta\left(x, x^{*}\right)}-\mathbf{1}\right\rangle\right)+\rho\left(x, x^{*}\right)\left\|\theta\left(x, x^{*}\right)\right\|^{2} \geq 0 \\
\Rightarrow & b\left(x, x^{*}\right)\left(\frac{1}{\tilde{r}}\left(e^{\tilde{r}\left[f(x)-f\left(x^{*}\right)\right]}-1\right)\right) \geq 0 \text { for } \tilde{p} \neq 0 \text { and } \tilde{r} \neq 0 .
\end{aligned}
$$

\section{Example 2.3}

(Zalmai [41]) The function $f$ is said to be first order $B-(b, \rho, \eta, \theta, \tilde{p}, \tilde{r})$-pseudoinvex with respect to $\eta$ and $b$ at $x^{*} \in X$ if there exist functions $\eta, \theta: X \times X \rightarrow \mathbb{R}^{n}$ and $b: X \times X \rightarrow[0, \infty)$, and real numbers $\tilde{r}$ and $\tilde{p}$ such that for all $x \in X$ and $z \in \mathbb{R}^{n}$,

$$
\begin{aligned}
& \frac{1}{\tilde{p}}\left(\left\langle\nabla f\left(x^{*}\right), e^{\tilde{p} \eta\left(x, x^{*}\right)}-\mathbf{1}\right\rangle\right)+\rho\left(x, x^{*}\right)\left\|\theta\left(x, x^{*}\right)\right\|^{2} \geq 0 \\
\Rightarrow \quad & b\left(x, x^{*}\right)\left(\frac{1}{\tilde{r}}\left(e^{\tilde{r}\left[f(x)-f\left(x^{*}\right)\right]}-1\right)\right) \geq 0 \text { for } \tilde{p} \neq 0 \text { and } \tilde{r} \neq 0 .
\end{aligned}
$$

We shall use the following auxiliary results which are crucial to the overall development of the main results on hand.

\section{Lemma 2.1}

For each $x \in X$,

$$
\varphi(x) \equiv \max _{1 \leq i \leq p} \frac{f_{i}(x)}{g_{i}(x)}=\max _{u \in U} \frac{\sum_{i=1}^{p} u_{i} f_{i}(x)}{\sum_{i=1}^{p} u_{i} g_{i}(x)}
$$

Theorem 2.1

[28] Let $x^{*} \in \mathbb{F}$ and $\lambda^{*}=\max _{1 \leq i \leq p} f_{i}\left(x^{*}\right) / g_{i}\left(x^{*}\right)$, for each $i \in p$, let $f_{i}$ and $g_{i}$ be twice continuously differentiable at $x^{*}$, for each $j \in q$, let the function $z \rightarrow G_{j}(z, t)$ be twice continuously differentiable at $x^{*}$ for all $t \in T_{j}$, and for each $k \in \underline{r}$, let the function $z \rightarrow H_{k}(z, s)$ be twice continuously differentiable at $x^{*}$ for all $s \in S_{k}$. If $x^{*}$ is an optimal solution of $(\mathrm{P})$, if the second order generalized Abadie constraint qualification holds at $x^{*}$, and if for any critical direction $y$, the set cone

$$
\begin{aligned}
& \left\{\left(\nabla G_{j}\left(x^{*}, t\right),\left\langle y, \nabla^{2} G_{j}\left(x^{*}, t\right) y\right\rangle\right): t \in \hat{T}_{j}\left(x^{*}\right), j \in \underline{q}\right\} \\
+ & \operatorname{span}\left\{\left(\nabla H_{k}\left(x^{*}, s\right),\left\langle y, \nabla^{2} H_{k}\left(x^{*}, s\right) y\right\rangle\right): s \in S_{k}, k \in \underline{r}\right\}, \\
& \text { where } \hat{T}_{j}\left(x^{*}\right) \equiv\left\{t \in T_{j}: G_{j}\left(x^{*}, t\right)=0\right\},
\end{aligned}
$$

is closed, then there exist $u^{*} \in U \equiv\left\{u \in \mathbb{R}^{p}: u \geq 0, \sum_{i=1}^{p} u_{i}=1\right\}$ and integers $\nu_{0}^{*}$ and $\nu^{*}$, with $0 \leq \nu_{0}^{*} \leq$ $\nu^{*} \leq n+1$, such that there exist $\nu_{0}^{*}$ indices $j_{m}$, with $1 \leq j_{m} \leq q$, together with $\nu_{0}^{*}$ points $t^{m} \in \hat{T}_{j_{m}}\left(x^{*}\right), m \in$ $\underline{\nu_{0}^{*}}, \nu^{*}-\nu_{0}^{*}$ indices $k_{m}$, with $1 \leq k_{m} \leq r$, together with $\nu^{*}-\nu_{0}^{*}$ points $s^{m} \in S_{k_{m}}$ for $m \in \underline{\nu^{*}} \underline{\nu_{0}^{*}}$, and $\nu^{*}$ real 
numbers $v_{m}^{*}$, with $v_{m}^{*}>0$ for $m \in \underline{\nu_{0}^{*}}$, with the property that

$$
\begin{aligned}
& \sum_{i=1}^{p} u_{i}^{*}\left[\nabla f_{i}\left(x^{*}\right)-\lambda^{*}\left(\nabla g_{i}\left(x^{*}\right)\right]+\sum_{m=1}^{\nu_{0}^{*}} v_{m}^{*}\left[\nabla G_{j_{m}}\left(x^{*}, t^{m}\right)\right.\right. \\
&+ \sum_{m=\nu_{0}^{*}+1}^{\nu^{*}} v_{m}^{*} \nabla H_{k}\left(x^{*}, s^{m}\right)=0 \\
&\left\langle y,\left[\sum_{i=1}^{p} u_{i}^{*}\left[\nabla^{2} f_{i}\left(x^{*}\right)-\lambda^{*} \nabla^{2} g_{i}\left(x^{*}\right)\right]+\sum_{m=1}^{\nu_{0}^{*}} v_{m}^{*} \nabla^{2} G_{j_{m}}\left(x^{*}, t^{m}\right)\right.\right. \\
&\left.\left.+\quad \sum_{m=\nu_{0}^{*}+1}^{\nu^{*}} v_{m}^{*} \nabla^{2} H_{k}\left(x^{*}, s^{m}\right)\right] y\right\rangle \geq 0
\end{aligned}
$$

where $\hat{T}_{j_{m}}\left(x^{*}\right)=\left\{t \in T_{j_{m}}: G_{j_{m}}\left(x^{*}, t\right)=0\right\}, U=\left\{u \in \mathbb{R}^{p}: u \geq 0, \sum_{i=1}^{p} u_{i}=1\right\}$, and $\underline{\nu^{*}} \backslash \underline{\nu_{0}^{*}}$ is the complement of the set $\underline{\nu_{0}^{*}}$ relative to the set $\underline{\nu^{*}}$.

\section{Second Order sufficient optimality conditions}

This section deals with some parametric sufficient efficiency conditions for problem $(\mathrm{P})$ under the generalized frameworks of second order $B-(b, \rho, \eta, \omega, \theta, \tilde{p}, \tilde{r}, \tilde{s})$-invexities for generalized invex functions. We start with real-valued functions $E_{i}\left(., x^{*}, u^{*}\right)$ and $B_{j}(., v)$ defined by

$$
E_{i}\left(x, x^{*}, u^{*}\right)=u_{i}\left[f_{i}(x)-\left(\frac{f_{i}\left(x^{*}\right)}{g_{i}\left(x^{*}\right)}\right) g_{i}(x)\right], i \in\{1, \cdots, p\}
$$

and

$$
B_{j}(., v)=v_{j} H_{j}(x), j=1, \cdots, m
$$

\section{Theorem 3.1}

Let $x^{*} \in Q$, functions $f_{i}, g_{i}$ for $i \in\{1, \cdots, p\}$ with $\varphi\left(x^{*}\right)=\frac{f_{i}\left(x^{*}\right)}{g_{i}\left(x^{*}\right)} \geq 0, g_{i}\left(x^{*}\right)>0$ and $H_{j}$ for $j \in\{1, \cdots, m\}$ be twice continuously differentiable at $x^{*} \in Q$, and let there exist $u^{*} \in U=\left\{u \in \mathbb{R}^{p}: u>0, \Sigma_{i=1}^{p} u_{i}=1\right\}$ and $v^{*} \in \mathbb{R}_{+}^{m}$ such that

$$
\begin{gathered}
\sum_{i=1}^{p} u_{i}^{*}\left[\nabla f_{i}\left(x^{*}\right)-\left(\frac{f_{i}\left(x^{*}\right)}{g_{i}\left(x^{*}\right)}\right) \nabla g_{i}\left(x^{*}\right)\right]+\sum_{j=1}^{m} v_{j}^{*} \nabla H_{j}\left(x^{*}\right)=0, \\
\left\langle z,\left[\sum_{i=1}^{p} u_{i}^{*}\left[\nabla^{2} f_{i}\left(x^{*}\right)-\left(\frac{f_{i}\left(x^{*}\right)}{g_{i}\left(x^{*}\right)}\right) \nabla^{2} g_{i}\left(x^{*}\right)\right]+\sum_{j=1}^{m} v_{j}^{*} \nabla^{2} H_{j}\left(x^{*}\right)\right] z\right\rangle \geq 0,
\end{gathered}
$$

where $z \in \mathbb{R}^{n}$, and

$$
v_{j}^{*} H_{j}\left(x^{*}\right)=0, j \in\{1, \cdots, m\} .
$$

Suppose, in addition, that any one of the following assumptions holds: 
(i) $E_{i}\left(. ; x^{*}, u^{*}\right) \forall i \in\{1, \cdots, p\}$ are second order $B-(b, \rho, \eta, \omega, \theta, \tilde{p}, \tilde{r}, \tilde{s})$-pseudoinvex with respect to $\eta, \omega$ and $b$ at $x^{*} \in X$ if there exist functions $\eta, \omega, \theta: X \times X \rightarrow \mathbb{R}^{n}$ and $b: X \times X \rightarrow \mathbb{R}_{+}=[0, \infty)$, and real numbers $\tilde{r}, \tilde{s}$ and $\tilde{p}$ for all $x \in X$ and $z \in \mathbb{R}^{n}$ with $b\left(x, x^{*}\right)>0$; and $B_{j}\left(., v^{*}\right) \forall j \in\{1, \cdots, m\}$ are second order $B-(\bar{b}, \rho, \eta, \omega, \theta, \tilde{p}, \tilde{r}, \tilde{s})$-quasiinvex with respect to $\eta, \omega$ and $\bar{b}$ at $x^{*} \in X$ if there exist functions $\eta, \omega, \theta: X \times X \rightarrow \mathbb{R}^{n}$ and $\bar{b}: X \times X \rightarrow \mathbb{R}_{+}=[0, \infty)$, and real numbers $\tilde{r}, \tilde{s}$ and $\tilde{p}$ for all $x \in X, z \in \mathbb{R}^{n}$, and $\rho\left(x, x^{*}\right) \geq 0$.

(ii) $E_{i}\left(. ; x^{*}, u^{*}\right) \forall i \in\{1, \cdots, p\}$ are second order $B-\left(b, \rho_{1}, \eta, \omega, \theta, \tilde{p}, \tilde{r}, \tilde{s}\right)$-pseudoinvex with respect to $\eta$, $\omega$ and $b$ at $x^{*} \in X$ if there exist functions $\eta, \omega, \theta: X \times X \rightarrow \mathbb{R}^{n}$ and $b: X \times X \rightarrow \mathbb{R}_{+}=[0, \infty)$, and real numbers $\tilde{r}, \tilde{s}$ and $\tilde{p}$ for all $x \in X$ and $z \in \mathbb{R}^{n}$ with $b\left(x, x^{*}\right)>0$; and $B_{j}\left(., v^{*}\right) \forall j \in\{1, \cdots, m\}$ are second order $B-\left(\bar{b}, \rho_{2}, \eta, \omega, \theta, \tilde{p}, \tilde{r}, \tilde{s}\right)$-quasiinvex with respect to $\eta, \omega$ and $\bar{b}$ at $x^{*} \in X$ if there exist functions $\eta, \omega, \theta: X \times X \rightarrow \mathbb{R}^{n}$ and $\bar{b}: X \times X \rightarrow \mathbb{R}_{+}=[0, \infty)$, and real numbers $\tilde{r}, \tilde{s}$ and $\tilde{p}$ for all $x \in X, z \in \mathbb{R}^{n}$, and $\rho_{1}\left(x, x^{*}\right), \rho_{2}\left(x, x^{*}\right) \geq 0$ with $\rho_{2}\left(x, x^{*}\right) \geq \rho_{1}\left(x, x^{*}\right)$.

(iii) $E_{i}\left(. ; x^{*}, u^{*}\right) \quad \forall i \in\{1, \cdots, p\}$ are second order prestrictly $B-\left(b, \rho_{1}, \eta, \omega, \theta, \tilde{p}, \tilde{r}, \tilde{s}\right)$-pseudoinvex with respect to $\eta, \omega$ and $b$ at $x^{*} \in X$ if there exist functions $\eta, \omega, \theta: X \times X \rightarrow \mathbb{R}^{n}$ and $b: X \times X \rightarrow \mathbb{R}_{+}=[0, \infty)$, and real numbers $\tilde{r}, \tilde{s}$ and $\tilde{p}$ for all $x \in X$ and $z \in \mathbb{R}^{n}$ with $b\left(x, x^{*}\right)>0$; and $B_{j}\left(., v^{*}\right) \forall j \in\{1, \cdots, m\}$ are second order strictly $B-\left(\bar{b}, \rho_{2}, \eta, \omega, \theta, \tilde{p}, \tilde{r}, \tilde{s}\right)$-quasiinvex with respect to $\eta, \omega$ and $b$ at $x^{*} \in X$ if there exist functions $\eta, \omega, \theta: X \times X \rightarrow \mathbb{R}^{n}$ and $\bar{b}: X \times X \rightarrow \mathbb{R}_{+}=[0, \infty)$, and real numbers $\tilde{r}, \tilde{s}$ and $\tilde{p}$ for all $x \in X$, $z \in \mathbb{R}^{n}$, and $\rho_{1}\left(x, x^{*}\right), \rho_{2}\left(x, x^{*}\right) \geq 0$ with $\rho_{2}\left(x, x^{*}\right) \geq \rho_{1}\left(x, x^{*}\right)$.

(iv) $E_{i}\left(. ; x^{*}, u^{*}\right) \forall i \in\{1, \cdots, p\}$ are second order prestrictly $B-\left(b, \rho_{1}, \eta, \omega, \theta, \tilde{p}, \tilde{r}, \tilde{s}\right)$-quasiinvex with respect to $\eta, \omega$ and $b$ at $x^{*} \in X$ if there exist functions $\eta, \omega, \theta: X \times X \rightarrow \mathbb{R}^{n}$ and $b: X \times X \rightarrow \mathbb{R}_{+}=[0, \infty)$, and real numbers $\tilde{r}, \tilde{s}$ and $\tilde{p}$ for all $x \in X$ and $z \in \mathbb{R}^{n}$ with $b\left(x, x^{*}\right)>0$; and $B_{j}\left(., v^{*}\right) \forall j \in\{1, \cdots, m\}$ are second order strictly $B-\left(\bar{b}, \rho_{2}, \eta, \omega, \theta, \tilde{p}, \tilde{r}, \tilde{s}\right)$-pseudoinvex with respect to $\eta$ and $\bar{b}$ at $x^{*} \in X$ if there exist functions $\eta, \omega, \theta: X \times X \rightarrow \mathbb{R}^{n}$ and $\bar{b}: X \times X \rightarrow \mathbb{R}_{+}=[0, \infty)$, and real numbers $\tilde{r}, \tilde{s}$ and $\tilde{p}$ for all $x \in X$, $z \in \mathbb{R}^{n}$, and $\rho_{1}\left(x, x^{*}\right), \rho_{2}\left(x, x^{*}\right) \geq 0$ with $\rho_{2}\left(x, x^{*}\right) \geq \rho_{1}\left(x, x^{*}\right)$.

(v) For each $i \in\{1, \cdots, p\}, f_{i}$ is second order $B-\left(b, \rho_{1}, \eta, \omega, \theta, \tilde{p}, \tilde{r}, \tilde{s}\right)-$ invex and $-g_{i}$ is second order $B-\left(b, \rho_{2}, \eta, \omega, \theta, \tilde{p}, \tilde{r}, \tilde{s}\right)$-invex at $x^{*}$ with $b\left(x, x^{*}\right)>0 . H_{j}\left(., v^{*}\right) \quad \forall j \in\{1, \cdots, m\} \quad$ is $B-$ $\left(\bar{b}, \rho_{3}, \eta, \omega, \theta, \tilde{p}, \tilde{r}, \tilde{s}\right)$-quasi-invex at $x^{*}$, and $\sum_{j=1}^{m} v_{j}^{*} \rho_{3}+\rho^{*} \geq 0$ for $\rho^{*}=\Sigma_{i=1}^{p} u_{i}^{*}\left(\rho_{1}+\phi\left(x^{*}\right) \rho_{2}\right)$ and for $\phi\left(x^{*}\right)=\frac{f_{i}\left(x^{*}\right)}{g_{i}\left(x^{*}\right)}$.

Then $x^{*}$ is an optimal solution to $(\mathrm{P})$.

\section{Proof}

If (i) holds, and if $x \in Q$, then it follows from (3.1) and (3.2) that

$$
\begin{gathered}
\frac{1}{\tilde{p}}\left\langle\sum_{i=1}^{p} u_{i}^{*}\left[\nabla f_{i}\left(x^{*}\right)-\left(\frac{f_{i}\left(x^{*}\right)}{g_{i}\left(x^{*}\right)}\right) \nabla g_{i}\left(x^{*}\right)\right], e^{\tilde{p} \eta\left(x, x^{*}\right)}-\mathbf{1}\right\rangle \\
+\frac{1}{\tilde{p}}\left\langle\sum_{j=1}^{m} v_{j}^{*} \nabla H_{j}\left(x^{*}\right), e^{\tilde{p} \eta\left(x, x^{*}\right)}-\mathbf{1}\right\rangle=0 \forall x \in Q, \\
\frac{1}{2 \tilde{s}}\left\langle e^{\tilde{s} \omega\left(x, x^{*}\right)}-\mathbf{1},\left[\sum_{i=1}^{p} u_{i}^{*}\left[\nabla^{2} f_{i}\left(x^{*}\right)-\left(\frac{f_{i}\left(x^{*}\right)}{g_{i}\left(x^{*}\right)}\right) \nabla^{2} g_{i}\left(x^{*}\right)\right]+\sum_{j=1}^{m} v_{j}^{*} \nabla^{2} H_{j}\left(x^{*}\right)\right] z\right\rangle \geq 0 .
\end{gathered}
$$

Since $v^{*} \geq 0, x \in Q$ and (3.3) holds, we have

$$
\sum_{j=1}^{m} v_{j}^{*} H_{j}(x) \leq 0=\Sigma_{j=1}^{m} v_{j}^{*} H_{j}\left(x^{*}\right),
$$


and so

$$
\bar{b}\left(x, x^{*}\right)\left(\frac{1}{\tilde{r}}\left(e^{\tilde{r}\left[H_{j}(x)-H_{j}\left(x^{*}\right)\right]}-1\right)\right) \leq 0
$$

since $\tilde{r} \neq 0$ and $\bar{b}\left(x, x^{*}\right) \geq 0$ for all $x \in Q$. In light of the $B-(\bar{b}, \rho, \eta, \omega, \theta, \tilde{p}, \tilde{r}, \tilde{s})$-quasiinvexity of $B_{j}\left(., v^{*}\right)$ at $x^{*}$, it follows that

$$
\frac{1}{\tilde{p}}\left(\left\langle\nabla H_{j}\left(x^{*}\right), e^{\tilde{p} \eta\left(x, x^{*}\right)}-\mathbf{1}\right\rangle\right)+\frac{1}{\tilde{s}}\left(\frac{1}{2}\left\langle e^{\tilde{s} \omega\left(x, x^{*}\right)}-\mathbf{1}, \nabla^{2} H_{j}\left(x^{*}\right) z\right\rangle\right)+\rho\left(x, x^{*}\right)\left\|\theta\left(x, x^{*}\right)\right\|^{2} \leq 0,
$$

and hence,

$$
\begin{aligned}
& \frac{1}{\tilde{p}}\left(\sum_{j=1}^{m}\left\langle\nabla H_{j}\left(x^{*}\right), e^{\tilde{p} \eta\left(x, x^{*}\right)}-\mathbf{1}\right\rangle\right)+\frac{1}{\tilde{s}}\left(\frac{1}{2}\left\langle e^{\tilde{s} \omega\left(x, x^{*}\right)}-\mathbf{1}, \Sigma_{j=1}^{m} \nabla^{2} H_{j}\left(x^{*}\right) z\right\rangle\right) \\
+ & \rho\left(x, x^{*}\right)\left\|\theta\left(x, x^{*}\right)\right\|^{2} \leq 0 .
\end{aligned}
$$

It follows from (3.4), (3.5) and (3.6) that

$$
\begin{aligned}
& \frac{1}{\tilde{p}}\left(\left\langle\sum_{i=1}^{p} u_{i}^{*}\left[\nabla f_{i}\left(x^{*}\right)-\left(\frac{f_{i}\left(x^{*}\right)}{g_{i}\left(x^{*}\right)}\right) \nabla g_{i}\left(x^{*}\right)\right], e^{\tilde{p} \eta\left(x, x^{*}\right)}-\mathbf{1}\right\rangle\right) \\
+ & \frac{1}{\tilde{s}}\left(\frac { 1 } { 2 } \left\langlee^{\tilde{s} \omega\left(x, x^{*}\right)}-\mathbf{1},\right.\right. \\
& \left.\left.\sum_{i=1}^{p} u_{i}^{*}\left[\nabla^{2} f_{i}\left(x^{*}\right) z-\left(\frac{f_{i}\left(x^{*}\right)}{g_{i}\left(x^{*}\right)}\right) \nabla^{2} g_{i}\left(x^{*}\right) z\right]\right\rangle\right) \\
\geq & \rho\left(x, x^{*}\right)\left\|\theta\left(x, x^{*}\right)\right\|^{2} .
\end{aligned}
$$

Since $\rho\left(x, x^{*}\right) \geq 0$, applying $B-(b, \rho, \eta, \omega, \theta, \tilde{p}, \tilde{r}, \tilde{s})$ - pseudo-invexity at $x^{*}$ to (3.7), we have

$$
\frac{1}{\tilde{r}} b\left(x, x^{*}\right)\left(e^{\tilde{r}\left[E_{i}\left(x, x^{*}, u^{*}\right)-E_{i}\left(x^{*}, x^{*}, u^{*}\right)\right]}-1\right) \geq 0 .
$$

Since $b\left(x, x^{*}\right)>0,(3.8)$ implies

$$
\begin{aligned}
& \sum_{i=1}^{p} u_{i}^{*}\left[f_{i}(x)-\left(\frac{f_{i}\left(x^{*}\right)}{g_{i}\left(x^{*}\right)}\right) g_{i}(x)\right] \\
\geq & \left.\sum_{i=1}^{p} u_{i}^{*}\left[f_{i}\left(x^{*}\right)-\left(\frac{f_{i}\left(x^{*}\right)}{g_{i}\left(x^{*}\right)}\right) g_{i}\left(x^{*}\right)\right]\right) \\
= & 0 .
\end{aligned}
$$

Thus, we have

$$
\Sigma_{i=1}^{p} u_{i}^{*}\left[f_{i}(x)-\left(\frac{f_{i}\left(x^{*}\right)}{g_{i}\left(x^{*}\right)}\right) g_{i}(x)\right] \geq 0 .
$$

Since $u_{i}^{*}>0$ for each $i \in\{1, \cdots, p\}$, we conclude using Lemma 2.1 that

$$
\varphi(x)=\max _{1 \leq i \leq p} \frac{f_{i}(x)}{g_{i}(x)}=\max _{u \in U} \frac{\sum_{i=1}^{p} u_{i}^{*} f_{i}(x)}{\sum_{i=1}^{p} u_{i^{*}} g_{i}(x)} \geq \max _{u \in U} \frac{\sum_{i=1}^{p} u_{i}^{*} f_{i}\left(x^{*}\right)}{\sum_{i=1}^{p} u_{i^{*}} g_{i}\left(x^{*}\right)}=\varphi\left(x^{*}\right) .
$$

Since $x \in Q$ is arbitrary, $x^{*}$ is an optimal solution to $(\mathrm{P})$. 
The proof for (ii) is similar to that of (i), but we include for the sake of the completeness. If (ii) holds, and if $x \in Q$, then it follows from (3.1) and (3.2) that

$$
\begin{gathered}
\frac{1}{\tilde{p}}\left\langle\sum_{i=1}^{p} u_{i}^{*}\left[\nabla f_{i}\left(x^{*}\right)-\left(\frac{f_{i}\left(x^{*}\right)}{g_{i}\left(x^{*}\right)}\right) \nabla g_{i}\left(x^{*}\right)\right], e^{\tilde{p} \eta\left(x, x^{*}\right)}-\mathbf{1}\right\rangle \\
+\frac{1}{\tilde{p}}\left\langle\sum_{j=1}^{m} v_{j}^{*} \nabla H_{j}\left(x^{*}\right), e^{\tilde{p} \eta\left(x, x^{*}\right)}-\mathbf{1}\right\rangle=0 \forall x \in Q, \\
\frac{1}{\tilde{s}}\left\langle e^{\tilde{s} \omega\left(x, x^{*}\right)}-\mathbf{1},\left[\sum_{i=1}^{p} u_{i}^{*}\left[\nabla^{2} f_{i}\left(x^{*}\right)-\left(\frac{f_{i}\left(x^{*}\right)}{g_{i}\left(x^{*}\right)}\right) \nabla^{2} g_{i}\left(x^{*}\right)\right]+\sum_{j=1}^{m} v_{j}^{*} \nabla^{2} H_{j}\left(x^{*}\right)\right] z\right\rangle \geq 0 .
\end{gathered}
$$

Since $v^{*} \geq 0, x \in Q$ and (3.3) holds, we have

$$
\sum_{j=1}^{m} v_{j}^{*} H_{j}(x) \leq 0=\sum_{j=1}^{m} v_{j}^{*} H_{j}\left(x^{*}\right),
$$

and so

$$
b\left(x, x^{*}\right)\left(\frac{1}{\tilde{r}}\left(e^{\tilde{r}\left[H_{j}(x)-H_{j}\left(x^{*}\right)\right]}-1\right)\right) \leq 0
$$

since $\tilde{r} \neq 0$ and $\bar{b}\left(x, x^{*}\right) \geq 0$ for all $x \in Q$. In light of the $B-\left(b, \rho_{2}, \eta, \omega, \theta, \tilde{p}, \tilde{r}\right)$-quasiinvexity of $B_{j}\left(., v^{*}\right)$ at $x^{*}$, it follows that

$$
\frac{1}{\tilde{p}}\left(\left\langle\nabla H_{j}\left(x^{*}\right), e^{\tilde{p} \eta\left(x, x^{*}\right)}-\mathbf{1}\right\rangle\right)+\frac{1}{\tilde{s}}\left(\frac{1}{2}\left\langle e^{\tilde{s} \omega\left(x, x^{*}\right)}-\mathbf{1}, \nabla^{2} H_{j}\left(x^{*}\right) z\right\rangle\right)+\rho_{2}\left(x, x^{*}\right)\left\|\theta\left(x, x^{*}\right)\right\|^{2} \leq 0,
$$

and hence,

$$
\frac{1}{\tilde{p}}\left(\Sigma_{j=1}^{m}\left\langle\nabla H_{j}\left(x^{*}\right), e^{\tilde{p} \eta\left(x, x^{*}\right)}-\mathbf{1}\right\rangle\right)+\frac{1}{\tilde{s}}\left(\frac{1}{2}\left\langle e^{\tilde{s} \omega\left(x, x^{*}\right)}-\mathbf{1}, \Sigma_{j=1}^{M} \nabla^{2} H_{j}\left(x^{*}\right) z\right\rangle\right)+\rho_{2}\left(x, x^{*}\right)\left\|\theta\left(x, x^{*}\right)\right\|^{2} \leq 0 .
$$

It follows from (3.10), (3.11) and (3.12) that

$$
\begin{aligned}
& \frac{1}{\tilde{p}}\left(\left\langle\Sigma_{i=1}^{p} u_{i}^{*}\left[\nabla f_{i}\left(x^{*}\right)-\left(\frac{f_{i}\left(x^{*}\right)}{g_{i}\left(x^{*}\right)}\right) \nabla g_{i}\left(x^{*}\right)\right], e^{\tilde{p} \eta\left(x, x^{*}\right)}-\mathbf{1}\right\rangle\right) \\
+ & \frac{1}{\tilde{s}}\left(\frac{1}{2}\left\langle e^{\tilde{s} \omega\left(x, x^{*}\right)}-\mathbf{1}, \sum_{i=1}^{p} u_{i}^{*}\left[\nabla^{2} f_{i}\left(x^{*}\right) z-\left(\frac{f_{i}\left(x^{*}\right)}{g_{i}\left(x^{*}\right)}\right) \nabla^{2} g_{i}\left(x^{*}\right) z\right]\right\rangle\right) \\
\geq & \rho_{2}\left(x, x^{*}\right)\left\|\theta\left(x, x^{*}\right)\right\|^{2} .
\end{aligned}
$$

Since $\rho_{1}\left(x, x^{*}\right), \rho_{2}\left(x, x^{*}\right) \geq 0$ with $\rho_{2}\left(x, x^{*}\right) \geq \rho_{1}\left(x, x^{*}\right)$, applying $B-\left(b, \rho_{1}, \eta, \omega, \theta, \tilde{p}, \tilde{r}\right)-$ pseudo-invexity at $x^{*}$ to (3.13), we have

$$
\frac{1}{\tilde{r}} b\left(x, x^{*}\right)\left(e^{\tilde{r}\left[E_{i}\left(x, x^{*}, u^{*}\right)-E_{i}\left(x^{*}, x^{*}, u^{*}\right)\right]}-1\right) \geq 0 .
$$

Since $b\left(x, x^{*}\right)>0,(3.14)$ implies

$$
\begin{aligned}
& \sum_{i=1}^{p} u_{i}^{*}\left[f_{i}(x)-\left(\frac{f_{i}\left(x^{*}\right)}{g_{i}\left(x^{*}\right)}\right) g_{i}(x)\right] \\
\geq & \left.\sum_{i=1}^{p} u_{i}^{*}\left[f_{i}\left(x^{*}\right)-\left(\frac{f_{i}\left(x^{*}\right)}{g_{i}\left(x^{*}\right)}\right) g_{i}\left(x^{*}\right)\right]\right) \\
= & 0 .
\end{aligned}
$$


Thus, we have

$$
\sum_{i=1}^{p} u_{i}^{*}\left[f_{i}(x)-\left(\frac{f_{i}\left(x^{*}\right)}{g_{i}\left(x^{*}\right)}\right) g_{i}(x)\right] \geq 0 .
$$

Since $u_{i}^{*}>0$ for each $i \in\{1, \cdots, p\}$, we conclude using Lemma 2.1 that

$$
\varphi(x)=\max _{1 \leq i \leq p} \frac{f_{i}(x)}{g_{i}(x)}=\max _{u \in U} \frac{\sum_{i=1}^{p} u_{i}^{*} f_{i}(x)}{\sum_{i=1}^{p} u_{i^{*}} g_{i}(x)} \geq \max _{u \in U} \frac{\sum_{i=1}^{p} u_{i}^{*} f_{i}\left(x^{*}\right)}{\sum_{i=1}^{p} u_{i^{*}} g_{i}\left(x^{*}\right)}=\varphi\left(x^{*}\right) .
$$

Since $x \in Q$ is arbitrary, $x^{*}$ is an optimal solution to $(\mathrm{P})$.

Next, we start off the proof for (iii) as follows: if (iii) holds, and if $x \in Q$, then it follows from (3.1) and (3.2) that

$$
\begin{aligned}
& \frac{1}{\tilde{p}}\left\langle\sum_{i=1}^{p} u_{i}^{*}\left[\nabla f_{i}\left(x^{*}\right)-\left(\frac{f_{i}\left(x^{*}\right)}{g_{i}\left(x^{*}\right)}\right) \nabla g_{i}\left(x^{*}\right)\right], e^{\tilde{p} \eta\left(x, x^{*}\right)}-\mathbf{1}\right\rangle \\
+ & \frac{1}{\tilde{p}}\left\langle\sum_{j=1}^{m} v_{j}^{*} \nabla H_{j}\left(x^{*}\right), e^{\tilde{p} \eta\left(x, x^{*}\right)}-\mathbf{1}\right\rangle=0 \forall x \in Q,
\end{aligned}
$$

and

$$
\frac{1}{\tilde{s}}\left(\left\langle e^{\tilde{s} \omega\left(x, x^{*}\right)}-\mathbf{1},\left[\sum_{i=1}^{p} u_{i}^{*}\left[\nabla^{2} f_{i}\left(x^{*}\right)-\left(\frac{f_{i}\left(x^{*}\right)}{g_{i}\left(x^{*}\right)}\right) \nabla^{2} g_{i}\left(x^{*}\right)\right]+\sum_{j=1}^{m} v_{j}^{*} \nabla^{2} H_{j}\left(x^{*}\right)\right] z\right\rangle\right) \geq 0 .
$$

Since $v^{*} \geq 0, x \in Q$ and (3.3) holds, we have

$$
\sum_{j=1}^{m} v_{j}^{*} H_{j}(x) \leq 0=\Sigma_{j=1}^{m} v_{j}^{*} H_{j}\left(x^{*}\right),
$$

which implies

$$
b\left(x, x^{*}\right)\left(\frac{1}{\tilde{r}}\left(e^{\tilde{r}\left[H_{j}(x)-H_{j}\left(x^{*}\right)\right]}-1\right)\right) \leq 0 .
$$

Then, in light of the strict $B-(b, \rho, \eta, \theta, \tilde{p}, \tilde{r}, \tilde{s})$-quasi-invexity of $B_{j}\left(., v^{*}\right)$ at $x^{*}$, we have

$$
\frac{1}{\tilde{p}}\left(\left\langle\nabla H_{j}\left(x^{*}\right), e^{\tilde{p} \eta\left(x, x^{*}\right)}-\mathbf{1}\right\rangle\right)+\frac{1}{\tilde{s}}\left(\frac{1}{2}\left\langle e^{\tilde{s} \omega\left(x, x^{*}\right)}-\mathbf{1}, \nabla^{2} H_{j}\left(x^{*}\right) z\right\rangle\right)+\rho\left(x, x^{*}\right)\left\|\theta\left(x, x^{*}\right)\right\|^{2}<0 .
$$

It follows from (3.3), (3.16), (3.17) and (3.18) that

$$
\begin{aligned}
& \frac{1}{\tilde{p}}\left(\left\langle\Sigma_{i=1}^{p} u_{i}^{*}\left[\nabla f_{i}\left(x^{*}\right)-\left(\frac{f_{i}\left(x^{*}\right)}{g_{i}\left(x^{*}\right)}\right) \nabla g_{i}\left(x^{*}\right)\right], e^{\tilde{p} \eta\left(x, x^{*}\right)}-\mathbf{1}\right\rangle\right) \\
+ & \frac{1}{\tilde{s}}\left(\frac{1}{2}\left\langle e^{\tilde{s} \omega\left(x, x^{*}\right)}-\mathbf{1}, \sum_{i=1}^{p} u_{i}^{*}\left[\nabla^{2} f_{i}\left(x^{*}\right) z-\left(\frac{f_{i}\left(x^{*}\right)}{g_{i}\left(x^{*}\right)}\right) \nabla^{2} g_{i}\left(x^{*}\right) z\right]\right\rangle\right) \\
> & \rho\left(x, x^{*}\right)\left\|\theta\left(x, x^{*}\right)\right\|^{2} .
\end{aligned}
$$

As a result, since $\rho\left(x, x^{*}\right) \geq 0$, applying the prestrict $(b, \rho, \eta, \theta, \tilde{p}, \tilde{r}, \tilde{s})$-pseudo-invexity at $x^{*}$ to (3.19), we have

$$
\left(\Sigma_{i=1}^{p} u_{i}^{*}\left[f_{i}(x)-\left(\frac{f_{i}\left(x^{*}\right)}{g_{i}\left(g^{*}\right)}\right) g_{i}(x)\right]-\sum_{i=1}^{p} u_{i}^{*}\left[f_{i}\left(x^{*}\right)-\left(\frac{f_{i}\left(x^{*}\right)}{g_{i}\left(x^{*}\right)}\right) g_{i}\left(x^{*}\right)\right]\right) \geq 0
$$

which implies 


$$
\begin{aligned}
& \sum_{i=1}^{p} u_{i}^{*}\left[f_{i}(x)-\left(\frac{f_{i}\left(x^{*}\right)}{g_{i}\left(x^{*}\right)}\right) g_{i}(x)\right] \\
\geq & \left.\sum_{i=1}^{p} u_{i}^{*}\left[f_{i}\left(x^{*}\right)-\left(\frac{f_{i}\left(x^{*}\right)}{g_{i}\left(x^{*}\right)}\right) g_{i}\left(x^{*}\right)\right]\right) \\
= & 0 .
\end{aligned}
$$

Thus, we have

$$
\Sigma_{i=1}^{p} u_{i}^{*}\left[f_{i}(x)-\left(\frac{f_{i}\left(x^{*}\right)}{g_{i}\left(x^{*}\right)}\right) g_{i}(x)\right] \geq 0 .
$$

Since $u_{i}^{*}>0$ for each $i \in\{1, \cdots, p\}$, we conclude using Lemma 2.1 that

$$
\varphi(x)=\max _{1 \leq i \leq p} \frac{f_{i}(x)}{g_{i}(x)}=\max _{u \in U} \frac{\sum_{i=1}^{p} u_{i}^{*} f_{i}(x)}{\sum_{i=1}^{p} u_{i^{*}} g_{i}(x)} \geq \max _{u \in U} \frac{\sum_{i=1}^{p} u_{i}^{*} f_{i}\left(x^{*}\right)}{\sum_{i=1}^{p} u_{i^{*}} g_{i}\left(x^{*}\right)}=\varphi\left(x^{*}\right) .
$$

Since $x \in Q$ is arbitrary, $x^{*}$ is an optimal solution to $(\mathrm{P})$.

The proof applying (iv) is similar to that of (iii), but still we include it as follows: if $x \in Q$, then it follows from (3.1) and (3.2) that

$$
\begin{aligned}
& \frac{1}{\tilde{p}}\left\langle\sum_{i=1}^{p} u_{i}^{*}\left[\nabla f_{i}\left(x^{*}\right)-\left(\frac{f_{i}\left(x^{*}\right)}{g_{i}\left(x^{*}\right)}\right) \nabla g_{i}\left(x^{*}\right)\right], e^{\tilde{p} \eta\left(x, x^{*}\right)}-\mathbf{1}\right\rangle \\
+ & \frac{1}{\tilde{p}}\left\langle\sum_{j=1}^{m} v_{j}^{*} \nabla H_{j}\left(x^{*}\right), e^{\tilde{p} \eta\left(x, x^{*}\right)}-\mathbf{1}\right\rangle=0 \forall x \in Q,
\end{aligned}
$$

and

$$
\frac{1}{\tilde{s}}\left(\left\langle e^{\tilde{s} \omega\left(x, x^{*}\right)}-\mathbf{1},\left[\sum_{i=1}^{p} u_{i}^{*}\left[\nabla^{2} f_{i}\left(x^{*}\right)-\left(\frac{f_{i}\left(x^{*}\right)}{g_{i}\left(x^{*}\right)}\right) \nabla^{2} g_{i}\left(x^{*}\right)\right]+\sum_{j=1}^{m} v_{j}^{*} \nabla^{2} H_{j}\left(x^{*}\right)\right] z\right\rangle\right) \geq 0 .
$$

Since $v^{*} \geq 0, x \in Q$ and (3.3) holds, we have

$$
\sum_{j=1}^{m} v_{j}^{*} H_{j}(x) \leq 0=\sum_{j=1}^{m} v_{j}^{*} H_{j}\left(x^{*}\right),
$$

which implies

$$
\bar{b}\left(x, x^{*}\right)\left(\frac{1}{\tilde{r}}\left(e^{\tilde{r}\left[H_{j}(x)-H_{j}\left(x^{*}\right)\right]}-1\right)\right) \leq 0 .
$$

Then, in light of the equivalent form for the strict $B-(\bar{b}, \rho, \eta, \omega, \theta, \tilde{p}, \tilde{r}, \tilde{s})-$ pseudo-invexity of $B_{j}\left(., v^{*}\right)$ at $x^{*}$, we have

$$
\frac{1}{\tilde{p}}\left(\left\langle\nabla H_{j}\left(x^{*}\right), e^{\tilde{p} \eta\left(x, x^{*}\right)}-\mathbf{1}\right\rangle\right)+\frac{1}{2 \tilde{s}}\left(\left\langle e^{\tilde{s} \omega\left(x, x^{*}\right)}-\mathbf{1}, \nabla^{2} H_{j}\left(x^{*}\right) z\right\rangle\right)+\rho\left(x, x^{*}\right)\left\|\theta\left(x, x^{*}\right)\right\|^{2}<0 .
$$

It follows from (3.3), (3.21) and (3.22) that

$$
\begin{aligned}
& \frac{1}{\tilde{p}}\left(\left\langle\sum_{i=1}^{p} u_{i}^{*}\left[\nabla f_{i}\left(x^{*}\right)-\left(\frac{f_{i}\left(x^{*}\right)}{g_{i}\left(x^{*}\right)}\right) \nabla g_{i}\left(x^{*}\right)\right], e^{\tilde{p} \eta\left(x, x^{*}\right)}-1\right\rangle\right) \\
+ & \frac{1}{\tilde{s}}\left(\frac{1}{2}\left\langle e^{\tilde{s} \omega\left(x, x^{*}\right)}-\mathbf{1}, \sum_{i=1}^{p} u_{i}^{*}\left[\nabla^{2} f_{i}\left(x^{*}\right) z-\left(\frac{f_{i}\left(x^{*}\right)}{g_{i}\left(x^{*}\right)}\right) \nabla^{2} g_{i}\left(x^{*}\right) z\right]\right\rangle\right) \\
> & \rho\left(x, x^{*}\right)\left\|\theta\left(x, x^{*}\right)\right\|^{2} .
\end{aligned}
$$


As a result, since $\rho\left(x, x^{*}\right) \geq 0$, applying the the equivalent form for the prestrict $B-(b, \rho, \eta, \omega, \theta, \tilde{p}, \tilde{r}, \tilde{s})-$ quasiinvexity of $E_{i}\left(. ; x^{*}, u^{*}\right)$ at $x^{*}$ to (3.23), we have

$$
b\left(x, x^{*}\right)\left(\sum_{i=1}^{p} u_{i}^{*}\left[f_{i}(x)-\left(\frac{f_{i}\left(x^{*}\right)}{g_{i}\left(g^{*}\right)}\right) g_{i}(x)\right]-\sum_{i=1}^{p} u_{i}^{*}\left[f_{i}\left(x^{*}\right)-\left(\frac{f_{i}\left(x^{*}\right)}{g_{i}\left(x^{*}\right)}\right) g_{i}\left(x^{*}\right)\right]\right) \geq 0,
$$

which (since $b\left(x, x^{*}\right)>0$ ) implies that

$$
\begin{aligned}
& \sum_{i=1}^{p} u_{i}^{*}\left[f_{i}(x)-\left(\frac{f_{i}\left(x^{*}\right)}{g_{i}\left(x^{*}\right)}\right) g_{i}(x)\right] \\
\geq & \left.\Sigma_{i=1}^{p} u_{i}^{*}\left[f_{i}\left(x^{*}\right)-\left(\frac{f_{i}\left(x^{*}\right)}{g_{i}\left(x^{*}\right)}\right) g_{i}\left(x^{*}\right)\right]\right) \\
= & 0 .
\end{aligned}
$$

Thus, we have

$$
\sum_{i=1}^{p} u_{i}^{*}\left[f_{i}(x)-\left(\frac{f_{i}\left(x^{*}\right)}{g_{i}\left(x^{*}\right)}\right) g_{i}(x)\right] \geq 0
$$

Since $u_{i}^{*}>0$ for each $i \in\{1, \cdots, p\}$, we conclude using Lemma 2.1 that

$$
\varphi(x)=\max _{1 \leq i \leq p} \frac{f_{i}(x)}{g_{i}(x)}=\max _{u \in U} \frac{\sum_{i=1}^{p} u_{i}^{*} f_{i}(x)}{\sum_{i=1}^{p} u_{i^{*}} g_{i}(x)} \geq \max _{u \in U} \frac{\sum_{i=1}^{p} u_{i}^{*} f_{i}\left(x^{*}\right)}{\sum_{i=1}^{p} u_{i^{*}} g_{i}\left(x^{*}\right)}=\varphi\left(x^{*}\right) .
$$

Since $x \in Q$ is arbitrary, $x^{*}$ is an optimal solution to $(\mathrm{P})$.

Finally, we prove (v) as follows: since $x \in Q$, it follows that $H_{j}(x) \leq H_{j}\left(x^{*}\right)$, which implies $\left(H_{j}(x)-H_{j}\left(x^{*}\right)\right) \leq 0$.

Then applying the $B-\left(\bar{b}, \rho_{3}, \eta, \omega, \theta, \tilde{p}, \tilde{r}, \tilde{s}\right)$-quasi-invexity of $H_{j}$ at $x^{*}$ and $v^{*} \in R_{+}^{m}$, we have

$$
\begin{aligned}
& \frac{1}{\tilde{p}}\left(\left\langle\sum_{j=1}^{m} v_{j}^{*} \nabla H_{j}\left(x^{*}\right), e^{\tilde{p} \eta\left(x, x^{*}\right)}-\mathbf{1}\right\rangle\right) \\
& +\frac{1}{\tilde{s}}\left(\frac{1}{2}\left\langle e^{\tilde{s} \omega\left(x, x^{*}\right)}-\mathbf{1}, \sum_{j=1}^{m} v_{j}^{*} \nabla^{2} H_{j}\left(x^{*}\right) z\right\rangle\right) \\
& \leq-\sum_{j=1}^{m} v_{j}^{*} \rho_{3}\left\|\theta\left(x, x^{*}\right)\right\|^{2} .
\end{aligned}
$$

Since $u^{*} \geq 0$ and $\frac{f_{i}\left(x^{*}\right)}{g_{i}\left(x^{*}\right)} \geq 0$, it follows from $B-\left(b, \rho_{3}, \eta, \omega, \theta, \tilde{p}, \tilde{r}, \tilde{s}\right)$-invexity assumptions that 


$$
\begin{aligned}
& b\left(x, x^{*}\right) \frac{1}{\tilde{r}}\left(e^{\tilde{r} \Sigma_{i=1}^{p} u_{i}^{*}\left(\left[f_{i}(x)-\left(\frac{f_{i}\left(x^{*}\right)}{g_{i}\left(x^{*}\right)}\right) g_{i}(x)\right]-\left[f_{i}\left(x^{*}\right)-\left(\frac{f_{i}\left(x^{*}\right)}{g_{i}\left(x^{*}\right)}\right) g_{i}\left(x^{*}\right)\right]\right)}-1\right) \\
= & b\left(x, x^{*}\right) \frac{1}{\tilde{r}}\left(e^{\tilde{r} \Sigma_{i=1}^{p} u_{i}^{*}\left\{\left[f_{i}(x)-f_{i}\left(x^{*}\right)\right]-\left(\frac{f_{i}\left(x^{*}\right)}{g_{i}\left(x^{*}\right)}\right)\left[g_{i}(x)-g_{i}\left(x^{*}\right)\right]\right\}}-1\right) \\
\geq & \frac{1}{\tilde{p}}\left(\sum_{i=1}^{p} u_{i}^{*}\left\{\left\langle\nabla f_{i}\left(x^{*}\right)-\left(\frac{f_{i}\left(x^{*}\right)}{g_{i}\left(x^{*}\right)}\right) \nabla g_{i}\left(x^{*}\right), e^{\tilde{p} \eta\left(x, x^{*}\right)}-\mathbf{1}\right\rangle\right\}\right) \\
+ & \frac{1}{2 \tilde{s}}\left(\left\langle e^{\tilde{s} \omega\left(x, x^{*}\right)}-\mathbf{1}, \Sigma_{i=1}^{p} u_{i}^{*}\left[\nabla^{2} f_{i}\left(x^{*}\right) z-\left(\frac{f_{i}\left(x^{*}\right)}{g_{i}\left(x^{*}\right)}\right) \nabla^{2} g_{i}\left(x^{*}\right) z\right\rangle\right]\right) \\
+ & \sum_{i=1}^{p} u_{i}^{*}\left[\rho_{1}+\phi\left(x^{*}\right) \rho_{2}\right]\left\|\theta\left(x, x^{*}\right)\right\|^{2} \\
\geq & -\left[\frac{1}{\tilde{p}}\left\langle\sum_{j=1}^{m} v_{j}^{*} \nabla H_{j}\left(x^{*}\right), e^{\tilde{p} \eta\left(x, x^{*}\right)}-\mathbf{1}\right\rangle+\frac{1}{2 \tilde{s}}\left\langle e^{\tilde{s} \omega\left(x, x^{*}\right)}-\mathbf{1}, \Sigma_{j=1}^{m} v_{j}^{*} \nabla^{2} H_{j}\left(x^{*}\right) z\right\rangle\right] \\
+ & \sum_{i=1}^{p} u_{i}^{*}\left[\rho_{1}+\phi\left(x^{*}\right) \rho_{2}\right]\left\|\theta\left(x, x^{*}\right)\right\|^{2} \\
\geq & \left(\sum_{j=1}^{m} v_{j}^{*} \rho_{3}+\sum_{i=1}^{p} u_{i}^{*}\left[\rho_{1}+\phi\left(x^{*}\right) \rho_{2}\right]\right)\left\|\theta\left(x, x^{*}\right)\right\|^{2} \\
= & \left(\sum_{j=1}^{m} v_{j}^{*} \rho_{3}+\rho^{*}\right)\left\|\theta\left(x, x^{*}\right)\right\|^{2} \geq 0,
\end{aligned}
$$

where $\phi\left(x^{*}\right)=\frac{f_{i}\left(x^{*}\right)}{g_{i}\left(x^{*}\right)}$ and $\rho^{*}=\Sigma_{i=1}^{p} u_{i}^{*}\left(\rho_{1}+\phi\left(x^{*}\right) \rho_{2}\right)$.

We Remark that when functions $f_{i}, g_{i}$ and $H_{j}$ have first-order derivatives, the established results seem to be specialized to $B-(p, r)$-invexities frameworks introduced by Antczak [1, 2, 3] and later generalized and investigated by Zalmai [41], Zalmai and Zhang [42] and others.

\section{Theorem 3.2}

([41], Theorem 3.2) Let $x^{*} \in Q$. Let $f_{i}, g_{i}$ for $i \in\{1, \cdots, p\}$ with $\phi\left(x^{*}\right)=\frac{f_{i}\left(x^{*}\right)}{g_{i}\left(x^{*}\right)} \geq 0, g_{i}\left(x^{*}\right)>0$ and $H_{j}$ for $j \in\{1, \cdots, m\}$ be differentiable at $x^{*} \in Q$, and let there exist $u^{*} \in U=\left\{u \in \mathbb{R}^{p}: u>0, \Sigma_{i=1}^{p} u_{i}=1\right\}$ and $v^{*} \in \mathbb{R}_{+}^{m}$ such that

$$
\Sigma_{i=1}^{p} u_{i}^{*}\left[\nabla f_{i}\left(x^{*}\right)-\left(\frac{f_{i}\left(x^{*}\right)}{g_{i}\left(x^{*}\right)}\right) \nabla g_{i}\left(x^{*}\right)\right]+\sum_{j=1}^{m} v_{j}^{*} \nabla H_{j}\left(x^{*}\right)=0
$$

and

$$
v_{j}^{*} H_{j}\left(x^{*}\right)=0, j \in\{1, \cdots, m\} .
$$

Suppose, in addition, that any one of the following assumptions holds:

(i) $E_{i}\left(. ; x^{*}, u^{*}\right) \forall i \in\{1, \cdots, p\}$ are $B-(b, \rho, \eta, \theta, \tilde{p}, \tilde{r})$-pseudoinvex with respect to $\eta$ and $b$ at $x^{*} \in X$ if there exist a function $\eta: X \times X \rightarrow \mathbb{R}^{n}$, a function $b: X \times X \rightarrow[0, \infty)$, and real numbers $\tilde{r}$ and $\tilde{p}$ such that for all $x \in X$ and $z \in \mathbb{R}^{n}$ with $b\left(x, x^{*}\right)>0$, and $B_{j}\left(., v^{*}\right) \forall j \in\{1, \cdots, m\}$ are $B-(b, \rho, \eta, \theta, \tilde{p}, \tilde{r})$-quasiinvex with respect to $\eta$ and $b$ at $x^{*} \in X$ if there exist a function $\eta: X \times X \rightarrow \mathbb{R}^{n}$, a function $b: X \times X \rightarrow[0, \infty)$, and real numbers $\tilde{r}$ and $\tilde{p}$ such that for all $x \in X, z \in \mathbb{R}^{n}$, and $\rho\left(x, x^{*}\right) \geq 0$. 
(ii) $E_{i}\left(. ; x^{*}, u^{*}\right) \forall i \in\{1, \cdots, p\}$ are $B-\left(b, \eta, \rho_{1}, \theta, \tilde{p}, \tilde{r}\right)$-pseudoinvex with respect to $\eta$ and $b$ at $x^{*} \in X$ if there exist a function $\eta: X \times X \rightarrow \mathbb{R}^{n}$, a function $b: X \times X \rightarrow[0, \infty)$, and real numbers $\tilde{r}$ and $\tilde{p}$ such that for all $x \in X$ and $z \in \mathbb{R}^{n}$, and $B_{j}\left(., v^{*}\right) \forall j \in\{1, \cdots, m\}$ are $B-\left(b, \rho_{2}, \eta, \theta, \tilde{p}, \tilde{r}\right)$-quasiinvex with respect to $\eta$, and $b$ and at $x^{*} \in X$ if there exist a function $\eta: X \times X \rightarrow \mathbb{R}^{n}$, a function $b: X \times X \rightarrow(0, \infty)$, and real numbers $\tilde{r}$ and $\tilde{p}$ such that for all $x \in X, z \in \mathbb{R}^{n}$, and $\rho_{1}\left(x, x^{*}\right), \rho_{2}\left(x, x^{*}\right) \geq 0$ with $\rho_{2}\left(x, x^{*}\right) \geq \rho_{1}\left(x, x^{*}\right)$.

(iii) $E_{i}\left(. ; x^{*}, u^{*}\right) \quad \forall i \in\{1, \cdots, p\} \quad$ are prestrictly $B-(b, \rho, \eta, \theta, \tilde{p}, \tilde{r})$-pseudo-invex at $x^{*}$, and $B_{j}\left(., v^{*}\right)$ $\forall j \in\{1, \cdots, m\}$ are strictly $B-(b, \rho, \eta, \theta, \tilde{p}, \tilde{r})$-quasi-invex at $x^{*}$.

(iv) $E_{i}\left(. ; x^{*}, u^{*}\right) \forall i \in\{1, \cdots, p\}$ are strictly $B-(b, \rho, \eta, \theta, \tilde{p}, \tilde{r})$-pseudo-invex at $x^{*}$, and $B_{j}\left(., v^{*}\right) \forall j \in$ $\{1, \cdots, m\}$ are strictly $B-(b, \rho, \eta, \theta, \tilde{p}, \tilde{r})$-quasi-invex at $x^{*}$ with $\rho\left(x, x^{*}\right) \geq 0$.

(v) For each $i \in\{1, \cdots, p\}, f_{i}$ is $B-\left(b, \rho_{1}, \eta, \theta, \tilde{p}, \tilde{r}\right)$-invex and $-g_{i}$ is $B-\left(b, \rho_{2}, \eta, \theta\right)$-invex at $x^{*} . H_{j}\left(., v^{*}\right)$ $\forall j \in\{1, \cdots, m\}$ is $B-\left(\rho_{3}, \eta\right)$-quasi-invex at $x^{*}$, and $\sum_{j=1}^{m} v_{j}^{*} \rho_{3}+\rho^{*} \geq 0$ for $\rho^{*}=\Sigma_{i=1}^{p} u_{i}^{*}\left(\rho_{1}+\phi\left(x^{*}\right) \rho_{2}\right)$ and for $\phi\left(x^{*}\right)=\frac{f_{i}\left(x^{*}\right)}{g_{i}\left(x^{*}\right)}$.

Then $x^{*}$ is an efficient solution to $(\mathrm{P})$.

\section{Concluding Remarks}

We observe that the obtained results in this communication can be applied to multiobjective fractional subset programming problems with generalized invex functions, for instance, based on the work of Mishra et al. [16] and Verma [29] to the case of the $\epsilon$ - efficiency and weak $\epsilon$-efficiency conditions to minimax fractional programming problems involving n-set functions. Furthermore, the generalized invexity frameworks developed to this context can also be applied to duality models for a class of multiobjective control problems as well as to a new class of multitime multiobjective variational problems of minimizing a vector of functionals of curvilinear integral type models.

\section{REFERENCES}

1. T. Antczak, A class of B-( $p, r)$-invex functions and mathematical programming, J. Math. Anal. Appl. 268 (2003), 187 - 206.

2. T. Antczak, Generalized fractional minimax programming with B-( $p, r)$-invexity, Computers and Mathematics with Applications $\mathbf{5 6}$ (2008), $1505-1525$.

3. T. Antczak, Generalized B-( $p, r)$-invexity functions and nonlinear mathematical programming, Numer. Funct. Anal. Optim. 30 (2009), $1-22$.

4. A. Ben-Israel and B. Mond What is the invexity? Journal of Australian Mathematical Society Ser. B 28 (1986), 1 - 9.

5. C. Canning, R Canning, Absence of pure Nash equilibria in a class of co-ordination games, Statistics, Optimization \& Information Computing 1 (1) (2013), 1 - 7.

6. M.A Hanson, On sufficiency of the Kuhn-Tucker conditions, Journal of Mathematical Analysis and Applications 80 (1985), 545-550.

7. V. Jeyakumar, Strong and weak invexity in mathematical programming, Methods Oper. Res. 55 (1985), 109 - 125.

8. M.H. Kim, G.S. Kim and G.M. Lee, On $\epsilon$-optimality conditions for multiobjective fractional optimization problems, Fixed Point Theory \& Applications 2011:6 doi:10.1186/1687-1812-2011-6.

9. G.S. Kim and G.M. Lee, On $\epsilon$-optimality theorems for convex vector optimization problems, Journal of Nonlinear and Convex Analysis 12 (3) (2013), 473 - 482.

10. Q Li, A modified Fletcher-Reeves-type method for nonsmooth convex optimization, Statistics, Optimization \& Information Computing 2(3)(2014), 200 - 210.

11. O.L. Mangasarian, Second- and higher-order duality theorems in nonlinear programming, J. Math. Anal. Appl. 51 (1975), 607 - 620.

12. S.K. Mishra, Second order generalized invexity and duality in mathematical programming, Optimization 42 (1997), 51 - 69.

13. S.K. Mishra, Second order symmetric duality in mathematical programming with F-convexity, European J. Oper. Res. 127 (2000), $507-518$

14. S.K. Mishra and N. G. Rueda, Higher-order generalized invexity and duality in mathematical programming, J. Math. Anal. Appl. 247 (2000), $173-182$.

15. S.K. Mishra and N. G. Rueda, Second-order duality for nondifferential minimax programming involving generalized type I functions, J. Optim. Theory Appl. 130 (2006), 477 - 486. 
16. S.K. Mishra, M. Jaiswal and Pankaj, Optimality conditions for multiple objective fractional subset programming with invex and related non-convex functions, Communications on Applied Nonlinear Analysis 17 (3) (2010), 89-101.

17. B. Mond and T. Weir, Generalized convexity and higher-order duality, J. Math. Sci. 16-18 (1981-1983), 74 - 94.

18. B. Mond and J. Zhang, Duality for multiobjective programming involving second-order V-invex functions, in Proceedings of the Optimization Miniconference II (B. M. Glover and V. Jeyakumar, eds.), University of New South Wales, Sydney, Australia ,1997, pp. $89-100$.

19. B. Mond and J. Zhang, Higher order invexity and duality in mathematical programming, in Generalized Convexity, Generalized Monotonicity: Recent Results (J. P. Crouzeix, et al., eds.), Kluwer Academic Publishers, printed in the Netherlands, 1998 , pp. 357 372.

20. R.B. Patel, Second order duality in multiobjective fractional programming, Indian J. Math. 38 (1997), 39 - 46.

21. M.K. Srivastava and M. Bhatia, Symmetric duality for multiobjective programming using second order $(F, \rho)$-convexity, Opsearch 43 (2006), 274 - 295.

22. K.K. Srivastava and M. G. Govil, Second order duality for multiobjective programming involving $(F, \rho, \sigma)$-type I functions, Opsearch 37 (2000), $316-326$.

23. S.K. Suneja, C. S. Lalitha, and S. Khurana, Second order symmetric duality in multiobjective programming, European J. Oper. Res. 144 (2003), $492-500$.

24. R.U. Verma and G.J. Zalmai, Generalized parametric duality models in discrete minmax fractional programming based on second order optimality conditions, Submitted for publication.

25. R.U. Verma, Weak $\epsilon$ - efficiency conditions for multiobjective fractional programming, Applied Mathematics and Computation 219 (2013), $6819-6827$.

26. R.U. Verma, A generalization to Zalmai type second order univexities and applications to parametric duality models to discrete minimax fractional programming, Advances in Nonlinear Variational Inequalities 15 (2) (2012), 113 - 123.

27. R.U. Verma, Parametric duality models for multiobjective fractional programming basedd on new generation hybrid invexities, Journal of Applied Functional Analysis 10 (3-4)(2015), 234 - 253.

28. R.U. Verma, Generalized $(\mathcal{G}, b, \beta, \phi, h, \rho, \theta)$-Univexities with Applications to Parametric Duality Models for Discrete Minimax Fractional Programming, Transactions on Mathematical Programming and Applications 1 (1) (2013), 1 - 14.

29. R.U. Verma, New $\epsilon$-optimality conditions for multiobjective fractional subset programming problems, Transactions on Mathematical Programming and Applications 1 (1) (2013), 69 - 89.

30. R.U. Verma, Second order $(\Phi, \Psi, \rho, \eta, \theta)$-invexity frameworks and $\epsilon$ - efficiency conditions for multiobjective fractional programming, Theory and Applications of Mathematics \& Computer Science 2 (2)(2012), 31 - 47.

31. X.M. Yang, Second order symmetric duality for nonlinear programs, Opsearch 32 (1995), 205 - 209.

32. X.M. Yang, On second order symmetric duality in nondifferentiable multiobjective programming, J. Ind. Manag. Optim. 5 (2009), $697-703$.

33. X.M. Yang and S. H. Hou, Second-order symmetric duality in multiobjective programming, Appl. Math. Lett. 14 (2001), 587 - 592.

34. X.M. Yang, K.L. Teo and X.Q. Yang, Higher-order generalized convexity and duality in nondifferentiable multiobjective mathematical programming, J. Math. Anal. Appl. 297 (2004), 48 - 55.

35. X.M. Yang, X.Q. Yang and K.L. Teo, Nondifferentiable second order symmetric duality in mathematical programming with Fconvexity, European J. Oper. Res. 144 (2003), 554 - 559.

36. X.M. Yang, X.Q. Yang and K.L. Teo, Huard type second-order converse duality for nonlinear programming, Appl. Math. Lett. 18 (2005), $205-208$.

37. X.M. Yang, X.Q. Yang and K.L. Teo, Higher-order symmetric duality in multiobjective programming with invexity, J. Ind. Manag. Optim. 4 (2008), $385-391$.

38. X.M. Yang, X.Q. Yang K.L. Teo and S.H. Hou, Second order duality for nonlinear programming, Indian J. Pure Appl. Math. 35 (2004), $699-708$.

39. K. Yokoyama, Epsilon approximate solutions for multiobjective programming problems, Journal of Mathematical Analysis and Applications 203 (1) (1996), 142-149.

40. G.J. Zalmai, General parametric sufficient optimality conditions for discrete minmax fractional programming problems containing generalized $(\theta, \eta, \rho)$-V-invex functions and arbitrary norms Journal of Applied Mathematics \& Computing 23 (1-2) (2007), 1 - 23.

41. G.J. Zalmai, Hanson-Antczak-type generalized invex functions in semiinfinte minmax fractional programming, Part I: Sufficient optimality conditions, Communications on Applied Nonlinear Analysis 19 (4) (2012), 1 - 36.

42. G.J. Zalmai and Q. Zhang, Global nonparametric sufficient optimality conditions for semiinfinite discrete minmax fractional programming problems involving generalized $(\eta, \rho)$-invex functions, Numerical Functional Analysis and Optimization 28 (2007), $173-209$.

43. J. Zhang and B. Mond, Second order b-invexity and duality in mathematical programming, Utilitas Math. 50 (1996), 19 - 31.

44. J. Zhang and B. Mond, Second order duality for multiobjective nonlinear programming involving generalized convexity, in Proceedings of the Optimization Miniconference III (B. M. Glover, B. D. Craven, and D. Ralph, eds.), University of Ballarat, (1997),pp. 79 - 95 . 